\title{
Solutions to a class of forced drift-diffusion equations with applications to the magneto-geostrophic equations
}

\author{
Susan Friedlander and Anthony Suen
}

\begin{abstract}
We prove the global existence of classical solutions to a class of forced drift-diffusion equations with $L^{2}$ initial data and divergence free drift velocity $\left\{u^{\nu}\right\}_{\nu>0} \subset L_{t}^{\infty} B M O_{x}^{-1}$, and we obtain strong convergence of solutions as the viscosity $\nu$ vanishes. We then apply our results to a family of active scalar equations which includes the three dimensional magneto-geostrophic $\left\{\mathrm{MG}^{\nu}\right\}_{\nu \geq 0}$ equation that has been proposed by Moffatt in the context of magnetostrophic turbulence in the Earth's fluid core. We prove the existence of a compact global attractor $\left\{\mathcal{A}^{\nu}\right\}_{\nu \geq 0}$ in $L^{2}\left(\mathbb{T}^{3}\right)$ for the $\mathrm{MG}^{\nu}$ equations including the critical equation where $\nu=0$. Furthermore, we obtain the upper semicontinuity of the global attractor as $\nu$ vanishes.
\end{abstract}

Mathematics Subject Classification (2010). 76D03, 35Q35, 76W05.

Keywords. active scalar equations, vanishing viscosity limit, global attractor.

\section{Introduction}

Our motivation for addressing the limiting behaviour of a class of drift diffusion equations comes from a model proposed by Moffatt and Loper [33, Moffatt [35] for magnetostrophic turbulence in the Earth's fluid core. This model is derived from the full three dimensional magnetohydrodynamic equations (MHD) in the context of a rapidly rotating, densely stratified, electrically conducting fluid. For discussions about the MHD equations in geophysical contexts see, for example, [1, 4], [17, [28], [34], 36]. Following the notation of Moffatt and Loper [33], we write the equations in terms of dimensionless variables. The orders of magnitude of the resulting non-dimensional parameters are motivated by the physical postulates of their model:

$$
\begin{aligned}
R_{0}\left(\partial_{t} u+u \cdot \nabla u\right)+e_{3} \times u & =-\nabla P+e_{2} \cdot \nabla b+R_{m} b \cdot \nabla b+\theta e_{3}+\nu \Delta u, \\
R_{m}\left[\partial_{t} b+u \cdot \nabla b-b \cdot \nabla u\right] & =e_{2} \cdot \nabla u+\Delta b, \\
\partial_{t} \theta+u \cdot \nabla \theta & =\kappa \Delta \theta+S, \\
\nabla \cdot u & =0, \nabla \cdot b=0 .
\end{aligned}
$$

The unknowns are $u(t, x)$ the velocity, $b(t, x)$ the magnetic field (both vector valued) and $\theta(t, x)$ the scalar (temperature field of the fluid). $P$ is the sum of the fluid and magnetic pressures, and the Cartesian unit vectors are given by $e_{1}, e_{2}$ and $e_{3}$. The physical forces governing this system are the Coriolis force, the Lorentz force and gravity acting via buoyancy, while the equation for the temperature is driven by a smooth function $S(x)$ that represents the external forcing of the MHD system.

The non-dimensional parameters in (1.1)-(1.4) are $R_{0}$ the Rossby number, $R_{m}$ the magnetic Reynolds number, $\nu$ a (non-dimensional) viscosity and $\kappa$ a (non-dimensional) thermal diffusivity. Moffatt and Loper argue that for the geophysical context they are modelling, all these parameters are 
small, with $\nu$ and $\kappa$ being extremely small. We note that the ratio of the Coriolis to Lorentz forces in their model is of order 1 , so for notational simplicity we have set this parameter, denoted by $N^{2}$ in [33], equal to 1. Hence in (1.1)-(1.4) we have a system derived from an important physical problem that is very rich in small parameters. The mathematical properties of this system under various settings of some of the parameters to zero, or in the vanishing limits have been addressed in a sequence of different articles, [19], 21], [22], [23], 24], 25], [26]. Although the physically relevant boundary for a model of the Earth's fluid core is a spherical annulus, for mathematical tractability these studies have considered the system on the periodic domain $\mathbb{T}^{3}$, with all fields being mean free, a condition which is preserved by the equations. In this present paper we study the forced system (1.1)-(1.4) under these boundary conditions.

The system as investigated by Moffatt and Loper neglects the terms multiplied by $R_{0}$ and $R_{m}$ in comparison with the remaining terms. Essentially this means that the evolution equations (1.1) and (1.2) for the coupled velocity and magnetic vectors take a simplified "quasi-static" form. A linear relationship is then established between the vector fields and the scalar temperature $\theta$. The sole remaining nonlinearity in the system occurs in the evolution equation for $\theta$ given by (1.3). This equation is then an advection-diffusion equation where the constitutive law that relates the divergence free velocity vector $u$ and the scalar $\theta$ is obtained from the reduced linear system

$$
\begin{aligned}
e_{3} \times u & =-\nabla P+e_{2} \cdot \nabla b+\theta e_{3}+\nu \Delta u, \\
0 & =e_{2} \cdot \nabla u+\Delta b, \\
\nabla \cdot u & =0, \nabla \cdot b=0 .
\end{aligned}
$$

This system encodes the vestiges of the physics in the problem, namely the Coriolis force, the Lorentz force and gravity. Vector manipulations of (1.5)-(1.7) give the expression

$$
\begin{aligned}
\left\{\left[\nu \Delta^{2}-\left(e_{2} \cdot \nabla\right)^{2}\right]^{2}+\left(e_{3} \cdot \nabla\right)^{2} \Delta\right\} u=-\left[\nu \Delta^{2}-\left(e_{2} \cdot \nabla\right)^{2}\right] \nabla & \times\left(e_{3} \times \nabla \theta\right) \\
& +\left(e_{3} \cdot \nabla\right) \Delta\left(e_{3} \times \nabla \theta\right) .
\end{aligned}
$$

We study the forced active scalar equation

$$
\left\{\begin{array}{l}
\partial_{t} \theta+u \cdot \nabla \theta=\kappa \Delta \theta+S \\
u=M^{\nu}[\theta], \theta(0, x)=\theta_{0}(x)
\end{array}\right.
$$

via an examination of the Fourier multiplier symbol of the operator $M^{\nu}$ obtained from (1.8). This active scalar equation is called the magnetogeostrophic (MG) equation. We also refer to (1.9) as the $\mathrm{MG}^{\nu}$ equation when $\nu>0$, and to the case when $\nu=0$ as the $\mathrm{MG}^{0}$ equation. In Section [5], we write the explicit expression for the Fourier multiplier symbol of $M^{\nu}$ obtained from (1.8). We observed that the limit $\nu \rightarrow 0$ is a highly singular limit: in particular, when $\nu>0$ the operator $M^{\nu}$ is smoothing of degree 2, however when $\nu=0$ the operator $M^{0}$ is singular of degree -1 . The goals of the current article are to examine the convergence of solutions to (1.9) in the limit as the viscosity $\nu$ goes to zero and to study the long time behaviour of the forced system.

Friedlander and Vicol [24] analyzed the unforced $S=0$ system (1.8)-(1.9) with the viscosity parameter $\nu$ set to zero, i.e. the unforced $\mathrm{MG}^{0}$ equation. In this situation the drift diffusion equation (1.9) is critical in the sense of the derivative balance between the advection and the diffusion term. They used De Giorgi techniques to obtain global well-posedness results for the unforced critical $\mathrm{MG}^{0}$ equation in a similar manner to the proof of global well-possedness given by Caffarelli and Vaseur [3] for the critical SQG equation. In Section 3 of this present paper we verify that the technical details of the De Giorgi techniques are, in fact, valid for drift diffusion equations with a smooth force. This procedure leads to the proof of Theorem 1.1, namely the existence of smooth classical solutions to the forced $\mathrm{MG}^{\nu}, \nu \geq 0$ equations. The existence of uniform $H^{s}$ bounds on these smooth solutions proved in Section 4 implies convergence of solutions as $\nu$ vanishes which is stated in Theorem 1.2

The second main result of this current paper concerns the existence of a global attractor for the critical $\mathrm{MG}^{0}$ equation. The issue of the existence of a global attractor for active scalar equations is important in the general context of the long time averages of solutions to forced fluid equations 
(c.f., [11]). In particular there are recent results concerning the existence of a global attractor for the dynamics of the forced critical SQG equation [8, [10, [12, [13]. In [8], Cheskidov and Dai prove that the forced critical SQG equation possesses a global attractor in $L^{2}\left(\mathbb{T}^{3}\right)$ provided the force $S$ is in $L^{p}\left(\mathbb{T}^{3}\right), p>2$. They use "classical" viscosity solutions and the abstract framework of evolutionary systems introduced by Cheskidov and Foias [6], 9]. We prove an analogous result for the forced three dimensional critical $\mathrm{MG}^{0}$ equation using the concept of a "vanishing viscosity" solution that arises naturally from the results of Theorem 1.2 concerning the convergence in the "vanishing viscosity" limit of solutions for the $\mathrm{MG}^{\nu}$ and $\mathrm{MG}^{0}$ equations. Our treatment of the $\mathrm{MG}^{\nu}, \nu \geq 0$, equations is novel in the sense that traditionally the existence of a global attractor is obtained from asymptotic compactness. For the MG equations the asymptotic compactness is not known a priori and the existence of the global attractor follows from

(a) the energy equality, which implies that the energy cannot grow rapidly (see Proposition 6.4),

(b) the absence of anomalous dissipation for complete bounded trajectories.

We note that the suggestion that this strategy might work for various equations was first proposed in 8.

The analysis in 24] of the unforced MG equations was given in the context of a class of drift diffusion equations where the divergence free drift velocity lies in $L_{t}^{\infty} B M O^{-1}$, which class includes the $\mathrm{MG}^{0}$ equation. We follow their approach using De Giorgi techniques to obtain global well-posedness results for a class of forced drift diffusion equations. More specifically, we study the following active scalar equation in $\mathbb{T}^{d} \times(0, \infty)$ with $d \geq 2$ :

$$
\left\{\begin{array}{l}
\partial_{t} \theta^{\nu}+u^{\nu} \cdot \nabla \theta^{\nu}=\kappa \Delta \theta^{\nu}+S \\
u_{j}^{\nu}=\partial_{i} T_{i j}^{\nu}\left[\theta^{\nu}\right], \theta(0, x)=\theta_{0}(x)
\end{array}\right.
$$

where $\nu \geq 0$. Here $\kappa>0$ is a diffusive constant, $\theta_{0}$ is the initial condition and $S=S(x)$ is a given smooth function that represents the forcing of the system. $\left\{T_{i j}^{\nu}\right\}_{\nu \geq 0}$ is a sequence of operators which satisfy:

(1.11) $\partial_{i} \partial_{j} T_{i j}^{\nu} f=0$ for any smooth functions $f$.

(1.12) $T_{i j}^{\nu}: L^{\infty}\left(\mathbb{T}^{d}\right) \rightarrow B M O\left(\mathbb{T}^{d}\right)$ are bounded for all $\nu \geq 0$.

(1.13) There exists a constant $C_{*}>0$ independent of $\nu$, such that for all $1 \leq i, j \leq d$,

$$
\begin{gathered}
\sup _{\nu \in(0,1]} \sup _{\left\{k \in \mathbb{Z}^{d}\right\}}\left|\widehat{T}_{i j}^{\nu}(k)\right| \leq C_{*} ; \\
\sup _{\left\{k \in \mathbb{Z}^{d}\right\}}\left|\widehat{T}_{i j}^{0}(k)\right| \leq C_{*},
\end{gathered}
$$

where $T_{i j}^{0}=\left.T_{i j}^{\nu}\right|_{\nu=0}$.

(1.14) For each $1 \leq i, j \leq d$,

$$
\lim _{\nu \rightarrow 0} \sum_{k \in \mathbb{Z}^{d}}\left|\widehat{T_{i j}^{\nu}}(k)-\widehat{T_{i j}^{0}}(k)\right|^{2}|\widehat{g}(k)|^{2}=0
$$

for all $g \in L^{2}$.

The main results that we prove for the forced problem are stated in the following theorems:

Theorem 1.1 (Existence of smooth solutions). Let $\theta_{0} \in L^{2}, S \in C^{\infty}$ and $\kappa>0$ be given, and assume that $\left\{T_{i j}^{\nu}\right\}_{\nu \geq 0}$ satisfy conditions (1.11)-(1.14). There exists a classical solution $\theta^{\nu}(t, x) \in C^{\infty}((0, \infty) \times$ $\mathbb{T}^{d}$ ) of (1.10), evolving from $\theta_{0}$ for all $\nu \geq 0$.

Theorem 1.2 (Convergence of solutions as $\nu \rightarrow 0$ ). Let $\theta_{0} \in L^{2}, S \in C^{\infty}$ and $\kappa>0$ be given, and assume that $\left\{T_{i j}^{\nu}\right\}_{\nu \geq 0}$ satisfy conditions (1.11)-(1.14). If $\theta^{\nu}, \theta$ are $C^{\infty}$ smooth classical solutions of the 
system (1.10) for $\nu>0$ and $\nu=0$ respectively with initial data $\theta_{0}$, then given $\tau>0$, for all $s \geq 0$, we have

$$
\lim _{\nu \rightarrow 0}\left\|\left(\theta^{\nu}-\theta\right)(t, \cdot)\right\|_{H^{s}}=0
$$

whenever $t \geq \tau$.

Theorem 1.3 (Existence of a global attractor for the MG equation). Let $\theta_{0} \in L^{2}, S \in C^{\infty}$ and $\kappa>0$ be given. The system (1.9) with $\nu=0$ possesses a compact global attractor $\mathcal{A}$ in $L^{2}\left(\mathbb{T}^{3}\right)$, namely

$$
\mathcal{A}=\left\{\theta_{0}: \theta_{0}=\theta(0) \text { for some bounded complete "vanishing viscosity" solution } \theta(t)\right\} .
$$

For any bounded set $\mathcal{B} \subset L^{2}\left(\mathbb{T}^{3}\right)$, and for any $\varepsilon, T>0$, there exists $t_{0}$ such that for any $t_{1}>t_{0}$, every "vanishing viscosity" solution $\theta(t)$ with $\theta(0) \in \mathcal{B}$ satisfies

$$
\|\theta(t)-x(t)\|_{L^{2}}<\varepsilon, \forall t \in\left[t_{1}, t_{1}+T\right],
$$

for some complete trajectory $x(t)$ on the global attractor $(x(t) \in \mathcal{A}, \forall t \in(-\infty, \infty))$.

Furthermore, for $\nu \in[0,1]$, there exists a compact global attractor $\mathcal{A}^{\nu} \subset L^{2}$ for (1.9) such that $\mathcal{A}^{0}=\mathcal{A}$ and $\mathcal{A}^{\nu}$ is upper semicontinuous at $\nu=0$, which means that

$$
\sup _{\phi \in \mathcal{A}^{\nu}} \inf _{\psi \in \mathcal{A}}\|\phi-\psi\|_{L^{2}} \rightarrow 0 \text { as } \nu \rightarrow 0 .
$$

Our paper is organised as follows. In Section 2, we give some preliminaries and notations which will be used in later sections. In Section [3, we state and prove Theorem 1.1, namely the existence of a smooth solution to (1.10). In Section 4, we obtain a uniform $H^{s}$-bound on smooth solutions to (1.10) and prove Theorem 1.2. In Section 5 we show that the $\mathrm{MG}^{\nu}$ equation with $\nu \geq 0$ satisfies the general conditions formulated for the active scalar equation in (1.10)-(1.14). Hence Theorem 1.2 can be applied to prove convergence as $\nu \rightarrow 0$ of solutions of the subcritical $\mathrm{MG}^{\nu}$ equation to solutions of the critical $\mathrm{MG}^{0}$ equation. In Section 6, we introduce the concept of a "vanishing viscosity" weak solution of the $\mathrm{MG}^{0}$ equation and we prove that the forced critical $\mathrm{MG}^{0}$ equation possesses a compact global attractor in $L^{2}\left(\mathbb{T}^{3}\right)$ satisfying (1.16).

\section{Preliminaries and notations}

In this section, we give some preliminaries and notations which are useful in later sections (also refer to [16] for more detailed discussion).

Throughout this paper, we shall denote $L^{p}\left([0, \infty) ; L^{q}\left(\mathbb{T}^{d}\right)\right)$ by $L_{t}^{p} L_{x}^{q}$ for $1 \leq p, q \leq \infty$, and similarly for $L_{t}^{p} B M O_{x}$, etc. Also, $L_{t, x}^{p}(I \times A)=L^{p}\left(I ; L^{p}(A)\right)$ for any $I \subset \mathbb{R}$ and $A \subset \mathbb{T}^{d}$.

Let $\varphi$ be a smooth function valued in $[0,1]$ such that $\varphi$ is supported in the shell $\left\{\xi \in \mathbb{R}^{d}: \frac{1}{2} \leq|\xi| \leq 4\right\}$. Denote

$$
h_{j}(x)=\sum_{k \in \tilde{\mathbb{Z}}^{d}} \varphi\left(2^{-j} k\right) e^{i k \cdot x}, x \in \mathbb{T}^{d},
$$

where $\tilde{\mathbb{Z}}^{d}$ is the dual lattice associated to $\mathbb{T}^{d}$. For $j \in \mathbb{Z}$, we define the periodic dyadic blocks as follows:

$$
\Delta_{j} f(x)=\frac{1}{(2 \pi)^{d}} \int_{\mathbb{T}^{d}} h_{j}(y) f(x-y) d y, x \in \mathbb{T}^{d} .
$$

For $s \in \mathbb{R}$ and $1 \leq p, q \leq \infty$, the Besov norm for $B_{p, q}^{s}=B_{p, q}^{s}\left(\mathbb{T}^{d}\right)$ is defined as (summation over repeated indices is understood):

$$
\|f\|_{B_{p, q}^{s}}=\left\|2^{j s}\right\| \Delta_{j} f\left\|_{L^{p}}\right\|_{\ell^{q}(\mathbb{Z})} .
$$

We also recall the Chemin-Lerner space-time Besov space $\tilde{L}^{r}\left(I ; B_{p, q}^{s}\right)$, with norm given by

$$
\|f\|_{\tilde{L}^{r}\left(I ; B_{p, q}^{s}\right)}=\left\|2^{j s}\left(\int_{I}\left\|\Delta_{j} f(t, \cdot)\right\|_{L^{p}}^{r} d t\right)^{\frac{1}{r}}\right\|_{\ell^{q}(\mathbb{Z})},
$$


where $s \in \mathbb{R}, 1 \leq r, p, q \leq \infty$ and $I$ is a time interval.

We will make use of the following well-known embedding theorems in later sections (refer to [2] for more details).

Gagliardo-Nirenberg-Sobolev inequality: Assume that $1 \leq p \leq d$. There exists a constant $C>0$ depending only on $p$ and $d$, such that

$$
\|f\|_{L^{d p /(d-p)}} \leq C\|D f\|_{L^{p}}
$$

for all $f \in C_{0}^{1}\left(\mathbb{T}^{d}\right)$.

Gagliardo-Nirenberg interpolation inequality: Fix $1 \leq q, r \leq \infty$ and $m \in \mathbb{N}$. Suppose that $m, p, \gamma, j$ satisfy

$$
\frac{1}{p}=\frac{j}{d}+\left(\frac{1}{r}-\frac{m}{d}\right) \gamma+\frac{1-\gamma}{q}, \quad \frac{j}{m} \leq \gamma \leq 1,
$$

where $d$ is the dimension, then

$$
\left\|D^{j} f\right\|_{L^{p}} \leq C\left\|D^{m} f\right\|_{L^{r}}^{\gamma}\|f\|_{L^{q}}^{1-\gamma},
$$

where $C>0$ is a constant which depends only on $m, d, j, q, r, \gamma$.

Gagliardo-Nirenberg interpolation inequality for Sobolev space: Let $q, r \in(1, \infty]$ and $\sigma, s \in(0, \infty)$ with $\sigma<s$. There exists a positive dimensional constant $C$ such that

$$
\|f\|_{W^{\sigma, p}} \leq C\|f\|_{L^{q}}^{\gamma}\|f\|_{W^{s, r},}^{1-\gamma},
$$

with $\frac{1}{p}=\frac{\gamma}{q}+\frac{1-\gamma}{r}$ and $\gamma=1-\frac{\sigma}{s}$.

Besov Embedding Theorem: Let $s \in \mathbb{R}$. If $1 \leq p_{1} \leq p_{2} \leq \infty$ and $1 \leq r_{1} \leq r_{2} \leq \infty$, then

$$
B_{p_{1}, r_{1}}^{s} \hookrightarrow B_{p_{2}, r_{2}}^{s-d\left(\frac{1}{p_{1}}-\frac{1}{p_{2}}\right)} \text {. }
$$

\section{Existence of smooth solutions}

In this section, we prove the existence of smooth solutions to the forced non-linear problem (1.10). It can be stated as follows.

Theorem 3.1. Let $\theta_{0} \in L^{2}, S \in C^{\infty}$ and $\kappa>0$ be given, and assume that $\left\{T_{i j}^{\nu}\right\}_{\nu \geq 0}$ satisfy conditions (1.11)-(1.14). There exists a classical solution $\theta^{\nu}(t, x) \in C^{\infty}\left((0, \infty) \times \mathbb{T}^{d}\right)$ of (1.10), evolving from $\theta_{0}$ for all $\nu \geq 0$.

\section{The Linear Problem}

Theorem 3.1 can be proved by the similar method as given in 24] with modification for the presence of a forcing term $S$ in (1.10). Following the proof given in [24], we first consider the linear problem:

$$
\partial_{t} \theta+(v \cdot \nabla) \theta=\kappa \Delta \theta+S,
$$

where the velocity vector $v(t, x)=\left(v_{1}(t, x), \cdots, v_{d}(t, x)\right) \in L^{2}\left((0, \infty) \times \mathbb{T}^{d}\right)$ is given, and $(t, x) \in$ $[0, \infty) \times \mathbb{T}^{d}$. Additionally, let $v$ satisfies

$$
\partial_{j} v_{j}(t, x)=0
$$

in the sense of distributions. We express $v_{j}$ as

$$
v_{j}(t, x)=\partial_{j} \partial_{i} V_{i j}(t, x) \text { in }[0, \infty) \times \mathbb{T}^{d},
$$

and we denoted $V_{i j}=-(-\Delta)^{-1} \partial_{i} v_{j}$. The matrix $\left\{V_{i j}\right\}_{i, j=1}^{d}$ is given, and satisfies

$$
V_{i j} \in L^{\infty}\left((0, \infty) ; L^{2}\left(\mathbb{T}^{d}\right) \cap L^{2}\left((0, \infty) ; H^{1}\left(\mathbb{T}^{d}\right)\right)\right.
$$

for all $i, j \in\{1, \ldots, d\}$.

We first prove the following proposition for the existence of smooth solutions to (3.1)-(3.4). 
Proposition 3.2. Given $\theta_{0} \in L^{2}$ and $S \in C^{\infty}$, and assume that $\left\{V_{i j}\right\}$ satisfies (3.4). Let

$$
\theta \in L^{\infty}\left([0, \infty) ; L^{2}\left(\mathbb{T}^{d}\right)\right) \cap L^{2}\left((0, \infty) ; H^{1}\left(\mathbb{T}^{d}\right)\right)
$$

be a global weak solution of the initial value problem associated to (3.1)-(3.4). If additionally we have $V_{i j} \in L^{\infty}\left(\left[t_{0}, \infty\right) ; B M O\left(\mathbb{T}^{d}\right)\right)$ for all $i, j \in\{1, \ldots, d\}$ and some $t_{0}>0$, then there exists $\alpha>0$ such that $\theta \in C^{\alpha}\left(\left[t_{0}, \infty\right) \times \mathbb{T}^{d}\right)$.

Remark 3.3. Note that for divergence-free $v \in L_{t, x}^{2}$, the existence of a weak solution $\theta$ to (3.1)-(3.4) evolving from $\theta_{0} \in L^{2}$ is well-known (for example, see [37] where the more general case $v \in L_{l o c}^{1}$ is discussed, also [3] and references therein). Here $\theta$ is a weak solution to (3.1)-(3.4) in the sense that $\theta$ satisfies (3.1)-(3.4) in a distributional sense, that is, for any $\phi \in C_{0}^{\infty}\left((0, T) \times \mathbb{T}^{3}\right)$,

$$
-\int_{0}^{T}\left\langle\theta, \phi_{t}\right\rangle d t-\int_{0}^{T}\langle v \theta, \nabla \phi\rangle d t+\int_{0}^{T}\langle\nabla \theta, \nabla \phi\rangle d t=\left\langle\theta_{0}, \phi(0, x)\right\rangle+\int_{0}^{T}\langle S, \phi\rangle d t,
$$

where $\langle\cdot, \cdot\rangle$ is the standard $L^{2}$-inner product on $\mathbb{T}^{d}$.

Proof of Proposition 3.2. In view of Theorem 2.1 in 24, we prove Proposition 3.2 in the following steps. Throughout the proof, we assume $\kappa \equiv 1$ for simplicity.

Step 1: A weak solution to (3.1)-(3.4) is bounded for positive time. In other words, there exists a positive constant $C$ such that for all $t>0$,

$$
\|\theta(t, \cdot)\|_{L^{\infty}} \leq C\|S\|_{L^{\infty}}^{-\frac{d}{d+4}}\left(\left\|\theta_{0}\right\|_{L^{2}}+\|S\|_{L^{\infty}}\right)\left(1+t^{-\frac{d}{2}}\right) .
$$

Proof. It follows by the similar method in proving Lemma 2.3 as in [8]. First, for $t>0$, we have the energy inequalities

$$
\begin{gathered}
\|\theta(t, \cdot)\|_{L^{2}} \leq C\left\|\theta_{0}\right\|_{L^{2}}+t\|S\|_{L^{\infty}}, \\
\int_{0}^{t} \int|\nabla \theta(s, x)|^{2} d x d s \leq C\left[\left\|\theta_{0}\right\|_{L^{2}}^{2}+t\|S\|_{L^{\infty}}^{2}\right],
\end{gathered}
$$

And for $h>0$, we have the following level set energy inequality for the truncated function $(\theta-h)_{+}$:

$$
\int\left|\left(\theta\left(t_{2}, x\right)-h\right)_{+}\right|^{2} d x+2 \int_{t_{1}}^{t_{2}} \int|\nabla(\theta-h)|^{2} \leq \int\left|\left(\theta\left(t_{1}, x\right)-h\right)_{+}\right|^{2} d x+2 \int_{t_{1}}^{t_{2}} \int\left|S(\theta-h)_{+}\right|
$$

for all $0<t_{1}<t_{2}<\infty$.

Next, we apply De Giorgi iteration method based on (3.9). First we fix $t_{0}>0$ and define

$$
c_{n}=\sup _{t_{n} \leq t \leq t_{0}} \int\left|\theta_{n}\right|^{2}+2 \int_{t_{n}}^{\infty} \int\left|\nabla \theta_{n}\right|^{2},
$$

where $\theta_{n}=\left(\theta(t, \cdot)-h_{n}\right)_{+}, t_{n}=t_{0}-\frac{t_{0}}{2^{n}}, h_{n}=H-\frac{H}{2^{n}}$ and $H$ to be chosen later. Then we have

$$
c_{n} \leq \frac{2^{n+1}}{t_{0}} \int_{t_{n-1}}^{\infty} \int \theta_{n}^{2} \cdot \chi_{\left\{\theta_{n}>0\right\}}+2 \int_{t_{n-1}}^{\infty} \int\left|S \theta_{n}\right|,
$$

where $\chi$ is the characteristic function and

$$
\chi_{\left\{\theta_{n}>0\right\}} \leq \frac{2^{n}}{H} \theta_{n-1} .
$$


For the first term on the right side of (3.10), using Gagliardo-Nirenberg inequality (2.2), it can be estimated as follows.

$$
\begin{aligned}
\frac{2^{n+1}}{t_{0}} \int_{t_{n-1}}^{\infty} \int \theta_{n}^{2} \cdot \chi_{\left\{\theta_{n}>0\right\}} & \leq \frac{2^{n+1}}{t_{0}}\left(\frac{2^{n}}{H}\right)^{p-2} \int_{t_{n-1}}^{\infty} \int \theta_{n-1}^{p} \\
& \leq C \frac{2^{n(p-1)+1}}{t_{0} H^{p-2}} \int_{t_{n-1}}^{\infty}\left(\int\left|\theta_{n-1}\right|^{2}\right)^{\frac{p(1-\gamma)}{2}}\left(\int\left|\nabla \theta_{n-1}\right|^{2}\right)^{\frac{p \gamma}{2}} \\
& \leq \frac{C}{t_{0}} \cdot \frac{2^{n(p-1)+1}}{H^{p-2}} C_{n-1}^{\frac{p}{2}},
\end{aligned}
$$

where $\gamma=\frac{2}{p}, p=2\left(1+\frac{2}{d}\right)$ and $C>0$ is a dimensional constant independent of $n$. Similarly, the second term on the right side of (3.10) is bounded by

$$
\begin{aligned}
\int_{t_{n-1}}^{\infty} \int\left|S \theta_{n}\right| & \leq\|S\|_{L^{\infty}} \int_{t_{n-1}}^{\infty} \int\left|\theta_{n}\right| \cdot \chi_{\left\{\theta_{n}>0\right\}}^{p-1} \\
& \leq\|S\|_{L^{\infty}}\left(\frac{2^{n}}{H}\right)^{p-1} \int_{t_{n-1}}^{\infty} \int\left|\theta_{n-1}\right|^{p} \\
& \leq C\|S\|_{L^{\infty}}\left(\frac{2^{n}}{H}\right)^{p-1} \int_{t_{n-1}}^{\infty}\left(\int\left|\theta_{n-1}\right|^{2}\right)^{\frac{p(1-\gamma)}{2}}\left(\int\left|\nabla \theta_{n-1}\right|^{2}\right)^{\frac{p \gamma}{2}} \\
& \leq C\|S\|_{L^{\infty}} \frac{2^{n(p-1)}}{H^{p-1}} c_{n-1}^{\frac{p}{2}} .
\end{aligned}
$$

Hence we conclude from (3.10) that

$$
c_{n} \leq \frac{C}{t_{0}} \cdot \frac{2^{n(p-1)+1}}{H^{p-2}} C_{n-1}^{\frac{p}{2}}+C\|S\|_{L^{\infty}} \frac{2^{n(p-1)}}{H^{p-1}} c_{n-1}^{\frac{p}{2}} .
$$

We choose $H$ in (3.11) large enough so that

$$
H=C\left(\frac{c_{0}^{\frac{1}{2}}}{t^{\frac{d}{2}}}+\|S\|_{L^{\infty}}^{-\frac{d}{d+4}} c_{0}^{\frac{1}{2}}\right),
$$

then the nonlinear iteration inequality (3.11) implies that $c_{n}$ converges to 0 as $n \rightarrow \infty$.

Hence $\theta\left(x, t_{0}\right) \leq H$ for almost every $x \in \mathbb{T}^{d}$. Applying the same procedure to $-\theta$ gives a lower bound for $\theta$. To show that (3.6) holds, we need to estimate the term $c_{0}$. Using (3.8), we have

$$
c_{0} \leq C\left(\left\|\theta_{0}\right\|_{L^{2}}^{2}+t_{0}\|S\|_{L^{\infty}}^{2}\right) .
$$

We combine (3.13) with (3.12) to obtain, for $t \leq 1$,

$$
\theta(t, x) \leq C\left(\left\|\theta_{0}\right\|_{L^{2}}+\|S\|_{L^{\infty}}\right)\left(\|S\|_{L^{\infty}}^{-\frac{d}{d+4}}+t^{-\frac{d}{2}}\right) .
$$

On the other hand, we fix $t=1$ in (3.14) and shift it by $t-1$ in time for $t>1$. Since the equation (3.1) is autonomous, we obtain, for $t>1$,

$$
\theta(t, x) \leq C\left(\|\theta(t-1, \cdot)\|_{L^{2}}+\|S\|_{L^{\infty}}\right)\left(\|S\|_{L^{\infty}}^{-\frac{d}{d+4}}+1\right) .
$$

Using the energy inequality (3.7),

$$
\|\theta(t-1, \cdot)\|_{L^{2}}^{2} \leq C\left[e^{-(t-1)}\left\|\theta_{0}\right\|_{L^{2}}^{2}+\|S\|_{L^{\infty}}\right] .
$$

Combining the above with (3.15) and using the fact that $e^{-(t-1)} \leq \frac{1}{t}$ for $t>0$, we obtain, for $t>1$,

$$
\theta(t, x) \leq C\left(t^{-\frac{1}{2}}\left\|\theta_{0}\right\|_{L^{2}}+\|S\|_{L^{\infty}}\right)\left(\|S\|_{L^{\infty}}^{-\frac{d}{d+4}}+1\right) .
$$

Combining (3.14) and (3.16), we conclude that (3.6) holds for $t>0$. 
Step 2: Next, we show that $\theta$ satisfies the first energy inequality, namely for any $0<r<R$ and $h \in \mathbb{R}$, we have

$$
\begin{aligned}
& \left\|(\theta-h)_{+}\right\|_{L_{t}^{\infty} L_{x}^{2}\left(Q_{r}\right)}^{2}+\left\|\nabla(\theta-h)_{+}\right\|_{L_{t, x}^{2}\left(Q_{r}\right)}^{2} \\
& \leq \frac{C R}{(R-r)^{2}}\left\|(\theta-h)_{+}\right\|_{L_{t, x}^{2}\left(Q_{R}\right)}^{2-\frac{2}{d+2}}\left\|(\theta-h)_{+}\right\|_{L_{t, x}^{\infty}\left(Q_{R}\right)}^{\frac{2}{d+2}}+C R^{y}\|S\|_{L^{\infty}}^{2}\left|\{\theta>h\} \cap Q_{R}\right|^{1-\frac{1}{d+2}} \\
& \quad+\frac{C R^{\frac{y+1}{2}}\|S\|_{L^{\infty}}}{(R-r)}\left\|(\theta-h)_{+}\right\|_{L_{t, x}^{2}\left(Q_{R}\right)}\left|\{\theta>h\} \cap Q_{R}\right|^{\frac{1}{2}-\frac{1}{d+2}}
\end{aligned}
$$

where $y=\frac{2(d+2)}{d}+1, C=C\left(d,\left\|V_{i j}\right\|_{L_{t}^{\infty} B M O_{x}}\right)>0$ is a positive constant, and we have denoted $Q_{\rho}=\left[t_{0}-\rho, t_{0}\right] \times B_{\rho}\left(x_{0}\right)$ for $\rho>0$ and an arbitrary $\left(t_{0}, x_{0}\right) \in(0, \infty) \times \mathbb{T}^{d}$. Notice that by (3.6), the right side of (3.17) is finite.

Proof. We follow the method for proving Lemma 2.6 in [24] and the only difference here comes from the extra forcing term $S$. Fix $h>0$ and let $0<r<R$ be such that $\frac{t_{0}}{2}-R^{2}>0$. Define $\eta(t, x) \in C_{0}^{\infty}\left((0, \infty) \times \mathbb{T}^{d}\right)$ to be a smooth cutoff function such that

. $0 \leq \eta \leq 1$ in $(0, \infty) \times \mathbb{T}^{d}$

- $\eta \equiv 1$ in $Q_{r}\left(x_{0}, t_{0}\right)$ and $\eta \equiv 0$ in $\operatorname{cl}\left\{Q_{R}^{c}\left(x_{0}, t_{0}\right) \cap\left\{(t, x): t \leq t_{0}\right\}\right.$;

$\cdot|\nabla \eta| \leq \frac{C}{R-r},|\nabla \nabla \eta| \leq \frac{C}{(R-r)^{2}},\left|\partial_{t} \eta\right| \leq \frac{C}{(R-r)^{2}}$ in $Q_{R}\left(x_{0}, t_{0}\right) / Q_{r}\left(x_{0}, t_{0}\right)$,

for some positive constant $C$. Define $t_{1}=t_{0}-R^{2}$ and let $t_{2} \in\left[t_{0}-r^{2}, t_{0}\right]$ be arbitrary. Multiply (3.1) by $(\theta-h)_{+} \eta^{2}$ and then integrate on $\left[t_{1}, t_{2}\right] \times \mathbb{T}^{d}$ to obtain

$$
\begin{aligned}
\int_{t_{1}}^{t_{2}} \int \partial_{t}\left((\theta-h)_{+}^{2}\right) \eta^{2} d x d t & -2 \int_{t_{1}}^{t_{2}} \int \partial_{j j}(\theta-h)_{+}(\theta-h)_{+} \eta^{2} d x d t+\int_{t_{1}}^{t_{2}} \int \partial_{i} V_{i j} \partial_{j}\left((\theta-h)_{+}^{2}\right) \eta^{2} d x d t \\
& =\int_{t_{1}}^{t_{2}} \int(\theta-h)_{+} \eta^{2} S d x d t .
\end{aligned}
$$

From the estimates as shown in [24], it follows from (3.18) that

$$
\begin{aligned}
& \left\|(\theta-h)_{+}\right\|_{L_{t}^{\infty} L_{x}^{2}\left(Q_{r}\right)}^{2}+\left\|\nabla(\theta-h)_{+}\right\|_{L_{t, x}^{2}\left(Q_{r}\right)}^{2} \\
& \leq \frac{C R}{(R-r)^{2}}\left\|(\theta-h)_{+}\right\|_{L_{t, x}^{2}\left(Q_{R}\right)}^{2-\frac{2}{d+2}}\left\|(\theta-h)_{+}\right\|_{L_{t, x}^{\infty}\left(Q_{R}\right)}^{\frac{2}{d+2}}+\left|\int_{t_{1}}^{t_{2}} \int(\theta-h)_{+} \eta^{2} S d x d t\right| .
\end{aligned}
$$

Using the Gagliardo-Nirenberg-Sobolev inequality (2.1) for $\eta(\theta-h)_{+} \in H_{0}^{1}$, the second term on the right side of (3.19) can be bounded by

$$
\begin{aligned}
& \left|\int_{t_{1}}^{t_{2}} \int(\theta-h)_{+} \eta^{2} S d x d t\right| \leq\|S\|_{L^{\infty}}\left(\int_{t_{1}}^{t_{2}} \int\left|\eta(\theta-h)_{+}\right|^{\frac{2 d}{d-2}}\right)^{\frac{d-2}{2 d}}\left|\{\theta>h\} \cap Q_{R}\right|^{\frac{1}{2}+\frac{1}{d}} \\
& \leq C\|S\|_{L^{\infty}}\left(\iint_{Q_{R}}\left|\nabla(\eta(\theta-h))_{+}\right|^{2}\right)^{\frac{1}{2}}\left|\{\theta>h\} \cap Q_{R}\right|^{\frac{1}{2}+\frac{1}{d}} \\
& \leq C\|S\|_{L^{\infty}}\left(\iint_{Q_{R}}\left|\nabla(\theta-h)_{+}\right|^{2}\right)^{\frac{1}{2}}\left|\{\theta>h\} \cap Q_{R}\right|^{\frac{1}{2}-\frac{1}{2(d+2)}}\left|Q_{R}\right|^{\frac{1}{2(d+2)}+\frac{1}{d}} \\
& +\frac{C\|S\|_{L^{\infty}}}{(R-r)}\left(\iint_{Q_{R}}\left|(\theta-h)_{+}\right|^{2}\right)^{\frac{1}{2}}\left|\{\theta>h\} \cap Q_{R}\right|^{\frac{1}{2}-\frac{1}{d+2}}\left|Q_{R}\right|^{\frac{1}{d+2}+\frac{1}{d}} \\
& \leq C\|S\|_{L^{\infty}}\left(\iint_{Q_{R}}\left|\nabla(\theta-h)_{+}\right|^{2}\right)^{\frac{1}{2}}\left|\{\theta>h\} \cap Q_{R}\right|^{\frac{1}{2}-\frac{1}{2(d+2)}} R^{\frac{d+2}{d}+\frac{1}{2}} \\
& +\frac{C\|S\|_{L^{\infty}}}{(R-r)}\left(\iint_{Q_{R}}\left|(\theta-h)_{+}\right|^{2}\right)^{\frac{1}{2}}\left|\{\theta>h\} \cap Q_{R}\right|^{\frac{1}{2}-\frac{1}{d+2}} R^{\frac{d+2}{d}+1},
\end{aligned}
$$


and hence using Hölder's inequality, the term $\left(\iint_{Q_{R}}\left|\nabla(\theta-h)_{+}\right|^{2}\right)^{\frac{1}{2}}$ can be absorbed by the left side of (3.19) and hence (3.17) follows.

Step 3: We give an estimate on the supremum of $\theta$ on a half cylinder in terms of the supremum on the full cylinder. Assume that $h_{0} \leq \sup _{Q_{r_{0}}} \theta$, where $r_{0}>0$ is arbitrary, then we have

$$
\sup _{Q_{\frac{r_{0}}{2}}} \theta \leq h_{0}+C\left(\frac{\left|\left\{\theta>h_{0}\right\} \cap Q_{r_{0}}\right|^{\frac{1}{d+2}}}{r_{0}}\right)^{\frac{1}{2}}\left(\sup _{Q_{r_{0}}} \theta-h_{0}\right)
$$

for some positive constant $C=C\left(d,\left\|V_{i j}\right\|_{L_{t}^{\infty} B M O_{x}}\right)$.

Proof. To facilitate the proof, we first introduce the following notations:

- $A(h, r)=\{\theta>h\} \cap Q_{r}$,

- $a(h, r)=|A(h, r)|$,

- $b(h, r)=\left\|(\theta-h)_{+}\right\|_{L_{t, x}^{2}\left(Q_{r}\right)}^{2}$,

- $M(r)=\sup _{Q_{r}} \theta$,

- $m(r)=\inf _{Q_{r}} \theta$.

Let $0<r<R$ and $0<h<H$. By the definitions of $a$ and $b$, we have

$$
a(H, r) \leq \frac{b(h, r)}{(H-h)^{2}} .
$$

Following the proof of Lemma 2.10 in [24], using (3.21) and the first energy inequality (3.17) as proved in Step 2, we have

$$
\begin{aligned}
b(h, r) \leq & C a(h, r)^{\frac{2}{d+2}} \frac{R}{(R-r)^{2}} b(h, R)^{1-\frac{1}{d+2}}\left\|(\theta-h)_{+}\right\|_{L_{t, x}^{\infty}\left(Q_{R}\right)}^{\frac{2}{d+2}} \\
& +C a(h, r)^{\frac{2}{d+2}}\|S\|_{L^{\infty}}^{2} \frac{R^{y}}{(H-h)^{2}} b(h, R)^{1-\frac{1}{d+2}}(H-h)^{\frac{2}{d+2}} \\
& +C a(h, r)^{\frac{2}{d+2}}\|S\|_{L^{\infty}} \frac{R^{\frac{y+1}{2}}}{(H-h)(R-r)} b(h, R)^{1-\frac{1}{d+2}}(H-h)^{\frac{2}{d+2}} .
\end{aligned}
$$

By combining (3.21) and (3.22), we obtain

$$
\begin{aligned}
b(H, r) \leq & \frac{C R}{(H-h)^{\frac{4}{d+2}}(R-r)^{2}} b(h, R)^{1+\frac{1}{d+2}}\left\|(\theta-h)_{+}\right\|_{L_{t, x}^{\infty}}^{\frac{2}{d+2}}\left(Q_{R}\right) \\
& +\frac{C R^{y}\|S\|_{L^{\infty}}^{2}}{(H-h)^{\frac{4}{d+2}}(H-h)^{2}} b(h, R)^{1+\frac{1}{d+2}}(H-h)^{\frac{2}{d+2}} \\
& +\frac{C R^{\frac{y+1}{2}}\|S\|_{L^{\infty}}}{(H-h)^{\frac{4}{d+2}}(H-h)(R-r)} b(h, R)^{1+\frac{1}{d+2}}(H-h)^{\frac{2}{d+2}} .
\end{aligned}
$$

We now apply the De Giorgi iteration method based on (3.23). Let $r_{n}=\frac{r_{0}}{2}+\frac{r_{0}}{2^{n+1}}, h_{n}=h_{\infty}-\frac{\left(h_{\infty}-h_{0}\right)}{2^{n}}$, and $b_{n}=b\left(h_{n}, r_{n+1}\right)$, for all $n \geq 0$, where $r_{0}$ and $h_{0}$ are as given and $h_{\infty}>0$ is to be chosen later. By letting $H=h_{n+1}, h=h_{n}, r=r_{n+2}$, and $R=r_{n+1}$ in (3.23), we have

$$
b_{n+1} \leq\left[\frac{C\left(M\left(r_{0}\right)-h_{0}\right)^{\frac{2}{d+2}}}{\left(h_{\infty}-h_{0}\right)^{\frac{4}{d+2}} r_{0}}+\frac{C r_{0}^{y}\|S\|_{L^{\infty}}^{2}}{\left(h_{\infty}-h_{0}\right)^{\frac{2}{d+2}}+2}+\frac{C r_{0}^{\frac{y}{2}-1}\|S\|_{L^{\infty}}}{\left(h_{\infty}-h_{0}\right)^{\frac{2}{d+2}+1}}\right] 2^{n\left(2+\frac{4}{d+1}\right)} b_{n}^{1+\frac{1}{d+2}} .
$$

We let $B=2^{4+2(d+2)}$ and choose $h_{\infty}$ large enough so that

$$
\left[\frac{C\left(M\left(r_{0}\right)-h_{0}\right)^{\frac{2}{d+2}}}{\left(h_{\infty}-h_{0}\right)^{\frac{4}{d+2}} r_{0}}+\frac{C r_{0}^{y}\|S\|_{L^{\infty}}^{2}}{\left(h_{\infty}-h_{0}\right)^{\frac{2}{d+2}+2}}+\frac{C r_{0}^{\frac{y}{2}-1}\|S\|_{L^{\infty}}}{\left(h_{\infty}-h_{0}\right)^{\frac{2}{d+2}}+1}\right] b_{0}^{1+\frac{1}{d+2}} \leq \frac{1}{B}
$$


then by induction, we obtain from (3.24) that $b_{n} \leq \frac{b_{0}}{B^{n}}$ for all $n \in \mathbb{N}$. The rest follows from the argument given in 24] and we omit the details here.

Step 4: We have the following second energy inequality in controlling the possible growth of level sets of the solution: fix an arbitrary $x_{0} \in \mathbb{T}^{d}$, let $h \in \mathbb{R}, 0<r<R$, and $0<t_{1}<t_{2}$. Then we have

$$
\left\|\left(\theta\left(t_{2}, \cdot\right)-h\right)_{+}\right\|_{L^{2}\left(B_{r}\right)}^{2} \leq\left\|\left(\theta\left(t_{1}, \cdot\right)-h\right)_{+}\right\|_{L^{2}\left(B_{r}\right)}^{2}+\frac{C_{0} R^{d}\left(t_{2}-t_{1}\right)}{(R-r)^{2}}\left\|(\theta-h)_{+}\right\|_{L^{\infty}\left(\left(t_{1}, t_{2}\right) \times B_{R}\right)}^{2},
$$

where $C_{0}=C_{0}\left(d,\left\|V_{i j}\right\|_{L_{t}^{\infty} B M O_{x}},\|S\|_{L^{\infty}}\right)$ is a sufficiently large positive constant. Notice that by (3.6), the right side of (3.25) is finite.

Proof. Similar to the first energy inequality, we follow the method for proving Lemma 2.11 in [24] and the only difference here comes from the forcing term $S$. Given $h, r, R, t_{1}, t_{2}$, we define $\eta \in C_{0}^{\infty}$ to be a smooth cutoff function such that

. $0 \leq \eta \leq 1$;

- $\eta \equiv 1$ on $B_{r}$ and $\eta \equiv 0$ on $B_{R}^{c}$;

. $|\nabla \eta(x)| \leq \min \left\{\frac{C}{R-r}, \frac{C R}{(R-r)^{2}}\left\|(\theta-h)_{+}\right\|_{L^{\infty}\left(\left(t_{1}, t_{2}\right) \times B_{R}\right)}\right\}$ for all $x \in \mathbb{T}^{d}$,

for some positive constant $C$. Multiply (3.1) by $(\theta-h)_{+} \eta^{2}$ and integrate on $\left[t_{1}, t_{2}\right] \times \mathbb{T}^{d}$, it follows from the estimates given in 24 , that

$$
\begin{aligned}
\left\|\left(\theta\left(t_{2}, \cdot\right)-h\right)_{+}\right\|_{L^{2}\left(B_{r}\right)}^{2} \leq & \left\|\left(\theta\left(t_{1}, \cdot\right)-h\right)_{+}\right\|_{L^{2}\left(B_{r}\right)}^{2}+\frac{C R^{d}\left(t_{2}-t_{1}\right)}{(R-r)^{2}}\left\|(\theta-h)_{+}\right\|_{L^{\infty}\left(\left(t_{1}, t_{2}\right) \times B_{R}\right)}^{2} \\
+ & \left|\int_{t_{1}}^{t_{2}} \int(\theta-h)_{+} \eta^{2} S d x d t\right|
\end{aligned}
$$

where $C=C\left(d,\left\|V_{i j}\right\|_{L_{t}^{\infty} B M O_{x}}\right)$ is a positive constant. Using the Gagliardo-Nirenberg-Sobolev inequality (2.1) for $\eta \in C_{0}^{\infty}$, we bound the far right side of the above as follows.

$$
\begin{aligned}
& \left|\int_{t_{1}}^{t_{2}} \int(\theta-h)_{+} \eta^{2} S d x d t\right| \leq\left\|(\theta-h)_{+}\right\|_{L^{\infty}\left(\left(t_{1}, t_{2}\right) \times B_{R}\right)}\|S\|_{L^{\infty}}\left(t_{1}-t_{2}\right)\left(\int_{B_{R}}|\eta|^{\frac{2 d}{d-2}}\right)^{\frac{d-2}{2 d}}\left|B_{R}\right|^{\frac{1}{2}-\frac{1}{d}}
\end{aligned}
$$

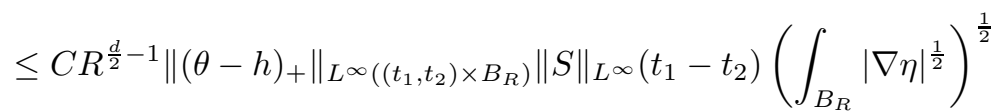

$$
\begin{aligned}
& \leq C R^{\frac{d}{2}-1+\frac{d}{2}}\left\|(\theta-h)_{+}\right\|_{L^{\infty}\left(\left(t_{1}, t_{2}\right) \times B_{R}\right)}^{2}\|S\|_{L^{\infty}}\left(t_{1}-t_{2}\right) \frac{C R}{(R-r)^{2}} \\
& =\frac{C\|S\|_{L^{\infty}} R^{d}\left(t_{2}-t_{1}\right)}{(R-r)^{2}}\left\|(\theta-h)_{+}\right\|_{L^{\infty}\left(\left(t_{1}, t_{2}\right) \times B_{R}\right)}^{2} .
\end{aligned}
$$

By using (3.27) on (3.26), we can choose $C_{0}=C_{0}\left(d,\left\|V_{i j}\right\|_{L_{t}^{\infty} B M O_{x}},\|S\|_{\infty}\right)>0$ sufficiently large enough so that (3.25) holds.

Step 5: Using the second energy inequality (3.25), we can bound $\left|\left\{\theta\left(t_{2}, \cdot\right) \geq H\right\} \cap B_{R}\right| /\left|B_{R}\right|$ whenever $\left|\left\{\theta\left(t_{1}, \cdot\right) \geq H\right\} \cap B_{r}\right| /\left|B_{r}\right| \leq \frac{1}{2}$. Fix $\kappa_{0}=\left(\frac{4}{5}\right)^{\frac{1}{d}}$, let $n_{0} \geq 2$ be the least integer such that $\frac{2^{n_{0}}}{2^{n_{0}-2}} \leq \sqrt{\frac{6}{5}}$, and let $\delta_{0}=\frac{\left(1-\kappa_{0}\right)^{2}}{12 C_{0} \kappa_{0}}$ where $C_{0}$ is the constant from (3.25). For $t_{1}, R>0$, if

$$
\left|\left\{\theta\left(t_{1}, \cdot\right) \geq H\right\} \cap B_{r}\right| \leq \frac{1}{2}\left|B_{r}\right|,
$$

then for all $t_{2} \in\left[t_{1}, t_{1}+\delta_{0} r^{2}\right]$ we have

$$
\left|\left\{\theta\left(t_{2}, \cdot\right) \geq H\right\} \cap B_{R}\right| \leq \frac{7}{8}\left|B_{R}\right|,
$$


where we define $r=r_{0} R, \quad M=\sup _{\left(t_{1}, t_{1}+\delta_{0} R^{2}\right) \times B_{R}} \theta, \quad m=\inf _{\left(t_{1}, t_{1}+\delta_{0} R^{2}\right) \times B_{R}} \theta, \quad h=\frac{(M+m)}{2}$ and $H=M-\frac{(M-m)}{2^{n_{0}}}$.

Proof. By using (3.25), the proof of (3.28) follows by the same argument given by the proof of Lemma 2.12 in $[24$ and we omit the details here.

Step 6: Applying Step 1 to Step 5, the proof of Proposition 3.2 now follows by showing that there exists $\beta \in(0,1)$ independent of $R$, such that

$$
\operatorname{osc}\left(Q_{1}\right) \leq \beta \operatorname{osc}\left(Q_{2}\right),
$$

where $Q_{1}=\left[t_{1}, t_{1}+\delta_{0} r^{2}\right] \times B_{r}, Q_{2}=\left[t_{1}, t_{1}+\delta_{0} R^{2}\right] \times B_{R}$ and $\operatorname{osc}(Q)=\sup _{Q} \theta-\inf _{Q} \theta$. Here $\kappa_{0}, \delta_{0}, n_{0}, M, m, h, H, r, R$ are defined as in Step 5, and we recall that $t_{1}>0$ and $R>0$ are arbitrary. We refer the reader to (24], pp. 293-294) for details in proving (3.29). The estimate (3.29) implies the Hölder regularity of the solution (the Hölder exponent $\alpha \in(0,1)$ may be calculated explicitly from $\beta$ ) which finishes the proof of Proposition 3.2.

\section{The Nonlinear Problem}

We now focus back on the non-linear problem (1.10) and give the proof of Theorem 3.1.

Proof of Theorem 3.1. To begin with, we notice that given $\theta_{0} \in L^{2}$ and $\nu \geq 0$, there exists a global-intime Leray-Hopf weak solution $\theta^{\nu}$ of (1.10) evolving from $\theta_{0}$ (a proof for it can be found in [24). Using the same method as in proving (3.6), we have $\theta^{\nu} \in L_{t, x}^{\infty}$ and it follows from the Caldern-Zygmund theory of singular integrals that $T_{i j}^{\nu} \theta=: V_{i j}^{\nu} \in L^{\infty}\left(\left[t_{0}, \infty\right) ; B M O\right)$, for any $t_{0}>0$ and $\nu \geq 0$, where $i, j \in\{1, \ldots, d\}$. Therefore, we may treat (1.10) as a linear evolution equation (see also [3, 15], 24]), where the divergence-free velocity field $u$ is given, and $u \in L^{2}\left((0, \infty) ; L^{2}\right) \cap L^{\infty}\left(\left[t_{0}, \infty\right) ; B M O^{-1}\right)$, for any $t_{0}>0$. This is precisely the setting of Proposition 3.2 for the linear evolution equation and it can be applied to the nonlinear problem (1.10) to give Hölder regularity of the solution. Finally, since Hölder regularity is sub-critical for the natural scaling of (1.10), one may bootstrap to prove that the solution is in a higher regularity class. We refer to 24 for further details and conclude the proof of Theorem 3.1

\section{Uniform bounds on smooth solutions and proof of Theorem 1.2}

\subsection{Uniform bounds on smooth solutions}

We have the following uniform $H^{s}$-bound on smooth solutions to (1.10) which will be used in proving Theorem [1.2.

Theorem 4.1. Assume that the hypotheses and notations of Theorem 3.1 are in force. Then given $0<t_{1}<t_{2}$ and $s \geq 0$, there exists a positive constant $C\left(C_{*}, t_{1}, t_{2}, s, d, \kappa, S,\left\|\theta_{0}\right\|_{L^{2}}\right)>0$ independent of $\nu$ such that

$$
\sup _{t \in\left[t_{1}, t_{2}\right]}\left\|\theta^{\nu}(t, \cdot)\right\|_{H^{s}}+\int_{t_{1}}^{t_{2}}\left\|\theta^{\nu}(t, \cdot)\right\|_{H^{s+1}}^{2} d t \leq C\left(C_{*}, t_{1}, t_{2}, s, d, \kappa, S,\left\|\theta_{0}\right\|_{L^{2}}\right),
$$

where $C_{*}>0$ is the constant as stated in condition (1.13).

Proof of Theorem 4.1. Fix $I=\left[t_{1}, t_{2}\right]$ for some $0<t_{1}<t_{2}$. By Theorem 3.1 there exists $\alpha \in(0,1)$ such that for each $\nu \geq 0$,

$$
\theta^{\nu} \in L^{\infty}\left(I ; L^{2}\left(\mathbb{T}^{d}\right) \cap L^{2}\left(I ; H^{1}\left(\mathbb{T}^{d}\right)\right) \cap L^{\infty}\left(I ; C^{\alpha}\left(\mathbb{T}^{d}\right)\right) .\right.
$$

Depending on the value of $\alpha$, we consider the following 2 cases:

Case 1: $\alpha \in\left(0, \frac{1}{2}\right]$. The proof is based on the one given in [26] and we just need to take extra care of the forcing term $S$. It is given in the following steps: 
Step (i): Assume further that

$$
\theta^{\nu} \in L^{2}\left(I ; B_{p, 2}^{1}\left(\mathbb{T}^{d}\right)\right)
$$

for some $p \geq 2$, then we have

$$
\theta^{\nu} \in \tilde{L}^{2}\left(I ; B_{q, r}^{1}\left(\mathbb{T}^{d}\right)\right)
$$

for all $1 \leq r \leq \infty$, and for all $q \in\left(p, m_{\alpha} p\right)$, where $m_{\alpha}=\frac{1-\alpha}{1-2 \alpha}>1$.

Proof. Let $\Delta_{j}$ be as defined in Section 2. We apply $\Delta_{j}$ to (1.10), multiply by $\Delta_{j} \theta\left|\Delta_{j} \theta\right|^{q-2}$, integrate over $\mathbb{T}^{d}$, and use Proposition 29.1 in 31 . (also refer to [5]) to obtain, for $j \in \mathbb{Z}$,

$$
\frac{1}{q} \frac{d}{d t}\left\|\Delta_{j} \theta^{\nu}\right\|_{L^{q}}^{q}+C 2^{2 j}\left\|\Delta_{j} \theta^{\nu}\right\|_{L^{q}}^{q} \leq\left.\left|\int \Delta_{j}\left(u^{\nu} \cdot \theta^{\nu}\right) \Delta_{j} \theta^{\nu}\right| \Delta_{j} \theta^{\nu}\right|^{q-2}|+| \int \Delta_{j}(S) \Delta_{j} \theta^{\nu}\left|\Delta_{j} \theta^{\nu}\right|^{q-2} \mid,
$$

where $C=C(d, q)>0$ is a positive constant independent of $\nu$. Using Hölder inequality, the second term on the right side of (4.5) is bounded by $\left\|\Delta_{j} \theta^{\nu}\right\|_{L^{q}}^{q-1}\left\|\Delta_{j}(S)\right\|_{L^{q}}$. Hence applying the similar estimates given in the proof of Lemma 2 in [26] pp. 259-261, we obtain

$$
\begin{aligned}
\frac{d}{d t}\left\|\Delta_{j} \theta^{\nu}\right\|_{L^{q}}+C 2^{2 j}\left\|\Delta_{j} \theta^{\nu}\right\|_{L^{q}} \leq & C\left\|\theta^{\nu}\right\|_{C^{\alpha}}^{2-\frac{p}{q}} 2^{j(1-\alpha)} \sum_{k \leq j} 2^{k\left(1-\frac{p}{q}-\alpha\left(1-\frac{p}{q}\right)\right)}\left(2^{k}\left\|\Delta_{k} \theta^{\nu}\right\|_{L^{p}}\right)^{\frac{p}{q}} \\
& +C\left\|\theta^{\nu}\right\|_{C^{\alpha}}^{2-\frac{p}{q}} 2^{j\left(2-\alpha-\frac{p}{q}-\alpha\left(1-\frac{p}{q}\right)\right)} \sum_{|j-k| \leq 2}\left(2^{k}\left\|\Delta_{k} \theta^{\nu}\right\|_{\left.L^{p}\right)^{\frac{p}{q}}}\right. \\
& +C\left\|\theta^{\nu}\right\|_{C^{\alpha}}^{2-\frac{p}{q}} 2^{j} \sum_{k \geq j-1} 2^{k\left(1-\alpha-\frac{p}{q}-\alpha\left(1-\frac{p}{q}\right)\right)}\left(2^{k}\left\|\Delta_{k} \theta^{\nu}\right\|_{L^{p}}\right)^{\frac{p}{q}} \\
& +C\left\|\Delta_{j} S\right\|_{L^{q}},
\end{aligned}
$$

where $C=C\left(C_{*}\right)>0$ is a positive constant independent of $\nu$ and $C_{*}$ is defined in condition (1.13). Applying Grönwall's inequality on (4.6),

$$
\begin{aligned}
\left\|\Delta_{j} \theta^{\nu}(t)\right\|_{L^{q}} \leq & e^{-c 2^{2 j\left(t-t_{1}\right)}}\left\|\Delta_{j} \theta^{\nu}\left(t_{1}\right)\right\|_{L^{q}} \\
& +C\left\|\theta^{\nu}\right\|_{L^{\infty}\left(I ; C^{\alpha}\right)}^{2-\frac{p}{q}} 2^{j(1-\alpha)} \sum_{k \leq j} 2^{k\left(1-\frac{p}{q}-\alpha\left(1-\frac{p}{q}\right)\right)} \Theta_{j, k}(t) \\
& +C\left\|\theta^{\nu}\right\|_{L^{\infty}\left(I ; C^{\alpha}\right)}^{2-\frac{p}{q}} 2^{j\left(2-\alpha-\frac{p}{q}-\alpha\left(1-\frac{p}{q}\right)\right)} \sum_{|k-j| \leq 2} \Theta_{j, k}(t) \\
& +C\left\|\theta^{\nu}\right\|_{L^{\infty}\left(I ; C^{\alpha}\right)}^{2-\frac{p}{q}} 2^{j} \sum_{k \geq j-1} 2^{k\left(1-\alpha-\frac{p}{q}-\alpha\left(1-\frac{p}{q}\right)\right)} \Theta_{j, k}(t) \\
& +\left\|\Delta_{j} S\right\|_{L^{q}} \int_{t_{1}}^{t} e^{-c(t-\tau) 2^{2 j}} d \tau,
\end{aligned}
$$

where

$$
\Theta_{j, k}(t)=\int_{t_{0}}^{t} e^{-c(t-\tau) 2^{2 j}}\left(2^{k}\left\|\Delta_{k} \theta^{\nu}(s)\right\|_{L^{p}}^{\frac{p}{q}}\right) d \tau
$$

We take the $L^{2}(I)$ norm of (4.7) and apply the similar estimates given in [26] pp. 260-261 to obtain

$$
\begin{aligned}
\left\|\Delta_{j} \theta^{\nu}(t)\right\|_{L^{2}\left(I ; L^{q}\right)} \leq & C\left\|\theta^{\nu}\left(t_{1}\right)\right\|_{L^{q}}^{\frac{p}{q}}\left\|\theta^{\nu}\left(t_{1}\right)\right\|_{C^{\alpha}}^{1-\frac{p}{q}}\left(2^{-j \alpha\left(1-\frac{p}{q}\right)} \min \left\{2^{-j},|I|^{\frac{1}{2}}\right\}\right) \\
& \left.+C\left\|\theta^{\nu}\right\|_{L^{\infty}\left(I ; C^{\alpha}\right)}\left\|\theta^{\nu}\right\|_{L^{2}\left(I ; B_{p, 2}^{1}\right)}^{\frac{p}{q}}|I|^{\frac{q-p}{2 q}}\left(2^{j\left(2-\alpha-\frac{p}{q}-\alpha\left(1-\frac{p}{q}\right)\right.}\right) \min \left\{C 2^{-2 j},|I|\right\}\right) \\
& +C\left\|\Delta_{j} S\right\|_{L^{q}|I|^{\frac{1}{2}} \min \left\{C 2^{-j},|I|\right\}}
\end{aligned}
$$


Multiply the above on both sides by $2^{j}$ and take an $\ell^{r}(\mathbb{Z})$-norm,

$$
\begin{aligned}
& \left\|\theta^{\nu}\right\|_{\tilde{L}^{2}\left(I ; B_{q, r}^{1}\right)} \leq C\left\|\theta^{\nu}\left(t_{1}\right)\right\|_{L^{q}}^{\frac{p}{q}}\left\|\theta^{\nu}\left(t_{1}\right)\right\|_{C^{\alpha}}^{1-\frac{p}{q}}\left\|\left(2^{j\left(1-\alpha\left(1-\frac{p}{q}\right)\right)} \min \left\{2^{-j},|I|^{\frac{1}{2}}\right\}\right)\right\|_{l^{r}(\mathbb{Z})} \\
& \left.+C\left\|\theta^{\nu}\right\|_{L^{\infty}\left(I ; C^{\alpha}\right)}\left\|\theta^{\nu}\right\|_{L^{2}\left(I ; B_{p, 2}^{1}\right)}^{\frac{p}{q}}|I|^{\frac{q-p}{2 q}} \|\left(2^{j\left(3-\alpha-\frac{p}{q}-\alpha\left(1-\frac{p}{q}\right)\right.}\right) \min \left\{C 2^{-2 j},|I|\right\}\right) \|_{l^{r}(\mathbb{Z})} \\
& +C\left\|2^{j}\right\| \Delta_{j} S\left\|_{L^{q}}|I|^{\frac{1}{2}} \min \left\{C 2^{-j},|I|\right\}\right\|_{\ell^{r}(\mathbb{Z})} .
\end{aligned}
$$

Since $q \in\left(p, m_{\alpha} p\right)$, the two $\ell^{r}$ norms on the right side of the above estimate are finite for any $1 \leq r \leq \infty$. On the other hand, the last term on the right side of (4.9) can be bounded by $\|S\|_{L^{\infty}}|I|^{\frac{1}{2}}$. Hence we have $\theta^{\nu} \in \tilde{L}^{2}\left(I ; B_{q, r}^{1}\right)$ which finishes the proof of (4.4).

Step (ii): Assume that $\theta^{\nu}$ satisfies (4.2), we then have

$$
\nabla \theta^{\nu} \in L^{2}\left(I ; L^{\infty}\left(\mathbb{T}^{d}\right)\right) .
$$

Proof. We follow the proof of Lemma 3 in [26] pp. 262-263. First, we note that $H^{1}=B_{2,2}^{1}$, so we may apply Step (i) with $p=2$ and obtain that $\theta \in L^{2}\left(I ; B_{q, 2}^{1}\right)$ for any $q \in\left(2,2 m_{\alpha}\right)$. Since $m_{\alpha}>1$, we may bootstrap and apply Step (i) once more to obtain that $\theta \in L^{2}\left(I ; B_{q, 2}^{1}\right)$ for all $q \in\left(2,2 m_{\alpha}^{2}\right)$. For any fixed $p>2$, we have $m_{\alpha}>1$ and hence $m_{\alpha}^{k} \rightarrow \infty$ as $k \rightarrow \infty$. By iterating Step (i) finitely many times, we obtain

$$
\theta^{\nu} \in \tilde{L}^{2}\left(I ; B_{p, r}^{1}\right) \text { for all } r \in[1, \infty]
$$

Fix $p$ large enough (which will be explicitly chosen later), and let $q=\frac{p\left(1+m_{\alpha}\right)}{2}$. From the estimate (4.8), for any $\varepsilon>0$,

$$
\begin{aligned}
& 2^{j(1+\varepsilon)}\left\|\Delta_{j} \theta^{\nu}(t)\right\|_{L^{2}\left(I ; L^{q}\right)} \leq C\left\|\theta^{\nu}\left(t_{1}\right)\right\|_{L^{q}}^{\frac{p}{q}}\left\|\theta^{\nu}\left(t_{1}\right)\right\|_{C^{\alpha}}^{1-\frac{p}{q}} \min \left\{C 2^{j\left(\varepsilon-\alpha\left(1-\frac{p}{q}\right)\right)},|I|^{\frac{1}{2}} 2^{j\left(1+\varepsilon-\alpha\left(1-\frac{p}{q}\right)\right)}\right\} \\
&+C\left\|\theta^{\nu}\right\|_{L^{\infty}\left(I ; C^{\alpha}\right)}\left\|\theta^{\nu}\right\|_{L^{2}\left(I ; B_{p, 2}^{1}\right)}^{\frac{p}{q}}|I|^{\frac{q-p}{2 q}} \\
&\left.\quad \times \min \left\{C 2^{j\left(\varepsilon+1-\frac{p}{q}-\alpha\left(2-\frac{p}{q}\right)\right.}, 2^{j\left(\varepsilon+3-\frac{p}{q}-\alpha\left(2-\frac{p}{q}\right)\right.}|I|\right\}\right) \\
&+C\left\|\Delta_{j} S\right\|_{L^{q}}|I|^{\frac{1}{2}} \min \left\{C 2^{j(\varepsilon-1)}, 2^{j(1+\varepsilon)}|I|\right\}
\end{aligned}
$$

Choose

$$
\varepsilon=\frac{1}{2} \min \left\{\frac{\alpha^{2}}{2-3 \alpha}, \frac{(1-2 \alpha)\left(2-3 \alpha-\alpha^{2}\right)}{(1-\alpha)(2-3 \alpha)}, 1\right\},
$$

then $\varepsilon>0$ for all $\alpha \in\left(0, \frac{1}{2}\right)$. By taking the $\ell^{r}$ norm of (4.12) and using Besov embedding theorem (2.4), we have

$$
\theta^{\nu} \in \tilde{L}^{2}\left(I ; B_{q, 1}^{1+\varepsilon}\right) \subset L^{2}\left(I ; B_{q, 1}^{1+\varepsilon}\right) \subset L^{2}\left(I ; B_{\infty, 1}^{1+\varepsilon-\frac{2 d}{p+p m_{\alpha}}}\right) .
$$

We pick $p>2$ so that $\varepsilon-\frac{2 d}{p+p m_{\alpha}}=0$, then we obtain from (4.13) that

$$
\nabla \theta^{\nu} \in L^{2}\left(I ; B_{\infty, 1}^{0}\right) \text {. }
$$

Lastly, from condition (4.2), we have $\nabla \theta^{\nu} \in L^{2}\left(I ; L^{2} \cap B_{\infty, 1}^{0}\right)$, hence (4.10) follows from the the borderline Sobolev embedding theorem that $L^{2} \cap B_{\infty, 1}^{0} \subset B_{\infty, 1}^{0} \subset L^{\infty}$.

Using the results obtained from Step (i) and Step (ii) as described above, we are ready to prove the bound (4.2) for $\alpha \in\left(0, \frac{1}{2}\right.$ ]. First, by using (4.10), we have

$$
\int_{t_{1}}^{t_{2}}\left\|\nabla \theta^{\nu}(t, \cdot)\right\|_{L^{\infty}}^{2} d t \leq C\left(C_{*}, t_{1}, t_{2}, d, \kappa, S,\left\|\theta_{0}\right\|_{L^{2}}\right),
$$


where $C\left(C_{*}, t_{1}, t_{2}, d, \kappa, S,\left\|\theta_{0}\right\|_{L^{2}}\right)>0$ is a positive constant independent of $\nu$. Next, by the condition $(1.2), u^{\nu}$ is divergence free and we have the a priori estimate derived from (1.10) that

$$
\begin{aligned}
& \frac{1}{2} \frac{d}{d t}\left\|\nabla \theta^{\nu}(t, \cdot)\right\|_{L^{2}}+\kappa\left\|\Delta \theta^{\nu}(t, \cdot)\right\|_{L^{2}} \\
& \leq\left|\int \partial_{k} u_{j}^{\nu} \partial_{k} \theta^{\nu} \partial_{j} \theta^{\nu}\right|+\left|\int S \Delta \theta^{\nu}\right| \\
& \leq C_{*}\left\|\Delta \theta^{\nu}(t, \cdot)\right\|_{L^{2}}\left\|\nabla \theta^{\nu}(t, \cdot)\right\|_{L^{2}}\left\|\nabla \theta^{\nu}(t, \cdot)\right\|_{L^{\infty}}+\left\|\nabla \theta^{\nu}(t, \cdot)\right\|_{L^{2}}\|\nabla S(t, \cdot)\|_{L^{2}} \\
& \leq \frac{\kappa}{2}\left\|\Delta \theta^{\nu}(t, \cdot)\right\|_{L^{2}}^{2}+C\left(C_{*}, \kappa, S\right)\left\|\nabla \theta^{\nu}(t, \cdot)\right\|_{L^{2}}^{2}\left\|\nabla \theta^{\nu}(t, \cdot)\right\|_{L^{\infty}}^{2} .
\end{aligned}
$$

By absorbing the term $\frac{\kappa}{2}\left\|\Delta \theta^{\nu}(t, \cdot)\right\|_{L^{2}}^{2}$ on the left side of (4.15) and using the bound (4.14), we obtain, for all $t \geq t_{1}$,

$$
\left\|\theta^{\nu}(t, \cdot)\right\|_{H^{1}}^{2} \leq\left\|\theta^{\nu}\left(t_{2}, \cdot\right)\right\|_{H^{1}}^{2} e^{\int_{t_{1}}^{t_{2}} C\left(C_{*}, \kappa, S\right)\left\|\nabla \theta^{\nu}(t, \cdot)\right\|_{L^{\infty}}^{2} d t} \leq\left\|\theta^{\nu}\left(t_{2}, \cdot\right)\right\|_{H^{1}}^{2} e^{C\left(C_{*}, t_{1}, t_{2}, d, \kappa, S,\left\|\theta_{0}\right\|_{L^{2}}\right)} .
$$

Since $\theta^{\nu} \in L^{2}\left(\left[t_{1}, t_{2}\right] ; H^{1}\right)$ and $L^{2}$ functions are finite a.e., $\left\|\theta^{\nu}\left(t_{2}, \cdot\right)\right\|_{H^{1}}$ is finite for a.e. $t_{2}>0$, with bounds in terms of $\left\|\theta_{0}\right\|_{L^{2}}$ but independent of $\nu$. Hence (4.16) implies $\theta^{\nu} \in L^{\infty}\left(\left[t_{1}, t_{2}\right] ; H^{1}\right) \cap$ $L^{2}\left(\left[t_{1}, t_{2}\right] ; H^{2}\right)$ with

$$
\sup _{t \in\left[t_{1}, t_{2}\right]}\left\|\theta^{\nu}(t, \cdot)\right\|_{H^{1}}+\int_{t_{1}}^{t_{2}}\left\|\theta^{\nu}(t, \cdot)\right\|_{\left.H^{2}\left(\mathbb{R}^{d}\right)\right)}^{2} d t \leq C\left(C_{*}, t_{1}, t_{2}, d, \kappa, S,\left\|\theta_{0}\right\|_{L^{2}}\right) .
$$

By further taking derivatives of the equation (1.10) and repeating the above argument, (4.1) also holds for all $s>1$ and we finish the proof of (4.1) for $\alpha \in\left(0, \frac{1}{2}\right]$.

Case 2: $\alpha \in\left(\frac{1}{2}, 1\right)$. We adopt the method given in [26] pp. 257-258. Similar to Case 1, the goal is to prove that (4.10) holds for $\theta^{\nu}$. Once (4.10) is proved, same argument given in the previous case can then be applied which gives the bound (4.1). First, note that if $\theta^{\nu}$ satisfies (4.2), then $\theta^{\nu} \in L^{\infty}\left(\left[t_{0}, \infty\right) ; B_{p, \infty}^{\alpha_{p}}\right)$, where $\alpha_{p}=\left(1-\frac{2}{p}\right) \alpha$ and $p \in[2, \infty)$ is fixed and to be chosen later. Then, for $j \in \mathbb{Z}$ fixed, we have

$$
\frac{1}{p} \frac{d}{d t}\left\|\Delta_{j} \theta^{\nu}\right\|_{L^{p}}^{p}+C 2^{2 j}\left\|\Delta_{j} \theta^{\nu}\right\|_{L^{p}}^{p} \leq\left.\left|\int\right| \Delta_{j} \theta^{\nu}\right|^{p-2} \Delta_{j} \theta^{\nu} \Delta_{j}\left(u \cdot \nabla \theta^{\nu}\right)|+| \int \Delta_{j}(S) \Delta_{j} \theta^{\nu}\left|\Delta_{j} \theta^{\nu}\right|^{p-2} \mid .
$$

Using the Bony paraproduct decomposition and the method given in [26], the first term on the

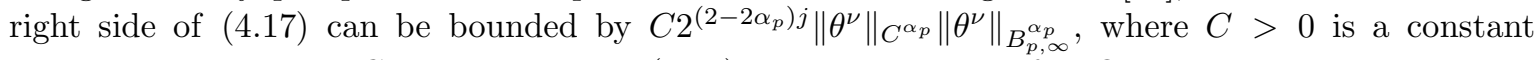
which may depend on $C_{*}$ as in condition (1.13) but independent of $\nu$. On the other hand, the term $\left.\left|\int \Delta_{j}(S) \Delta_{j} \theta^{\nu}\right| \Delta_{j} \theta^{\nu}\right|^{p-2} \mid$ can be bounded by $\left\|\Delta_{j} \theta^{\nu}\right\|_{L^{p}}^{p-1}\left\|\Delta_{j} S\right\|_{L^{p}}$. By applying the bounds on (4.17), using Gröwall inequality and the Besov embedding theorem (2.4), we obtain

$$
\theta^{\nu} \in L^{\infty}\left([t, \infty) ; B_{p, \infty}^{2 \alpha_{p}}\right) \subset L^{\infty}\left([t, \infty) ; B_{\infty, \infty}^{2 \alpha-\frac{4 \alpha+d}{p}}\right)
$$

for all $t \geq t_{1}$. Choose $p>\frac{4+d}{2 \alpha-1}$, then $2 \alpha-\frac{4 \alpha+d}{p}>1$. Since $L^{\infty} \cap B_{\infty, \infty}^{2 \alpha-\frac{4 \alpha+d}{p}}=C^{2 \alpha-\frac{4 \alpha+d}{p}}$, we conclude that (4.10) holds for $\theta^{\nu}$ and we finish the proof of (4.1) for $\alpha \in\left(\frac{1}{2}, 1\right)$.

\subsection{Proof of Theorem 1.2}

The proof can be divided into two cases:

Case 1: $s=0$. Let $\phi=\theta^{\nu}-\theta$, then $\phi$ satisfies the following equation:

$$
\partial_{t} \phi+u^{\nu} \cdot \nabla \phi+\left(u^{\nu}-u\right) \cdot \nabla \theta=\kappa \Delta \phi .
$$


Multiply (4.18) by $\phi$ and integrate,

$$
\frac{1}{2} \frac{d}{d t}\|\phi(t, \cdot)\|_{L^{2}}^{2}+\frac{\kappa}{2}\|\nabla \phi(t, \cdot)\|_{L^{2}}^{2}=-\int\left(u^{\nu}-u\right) \cdot \nabla \theta \cdot \phi(t, x) d x .
$$

We estimate the right side of (4.19) as follows. For each $t>0$,

$$
\begin{aligned}
\left|-\int\left(u^{\nu}-u\right) \cdot \nabla \theta \cdot \phi(t, x) d x\right| & \leq\left\|\left(u^{\nu}-u\right)(t, \cdot)\right\|_{L^{2}}\|\phi(t, \cdot)\|_{L^{2}}\|\nabla \theta(t, \cdot)\|_{L^{\infty}} \\
& \leq \frac{\kappa}{4 C_{*}^{2}}\left\|\left(u^{\nu}-u\right)(t, \cdot)\right\|_{L^{2}}^{2}+\frac{4 C_{*}^{2}}{\kappa}\|\phi(t, \cdot)\|_{L^{2}}^{2}\|\nabla \theta(t, \cdot)\|_{L^{\infty}}^{2},
\end{aligned}
$$

where $C_{*}>0$ is the constant as stated in condition (1.13). We focus on the term $\left\|\left(u^{\nu}-u\right)(t, \cdot)\right\|_{L^{2}}^{2}$ as in (4.20), and for simplicity we sometime drop the variable $t$. Using Plancherel Theorem, for each $j$,

$$
\begin{aligned}
\left\|\left(u_{j}^{\nu}-u_{j}\right)(t, \cdot)\right\|_{L^{2}}^{2} & =\sum_{k \in \mathbb{Z}^{d}}\left|\left(\widehat{u_{j}^{\nu}-u_{j}}\right)(k)\right|^{2} \\
& \left.=\sum_{k \in \mathbb{Z}^{d}} \mid \widehat{\partial_{i} T_{i j}^{\nu}} \widehat{\theta^{\nu}}-\widehat{\partial_{i} T_{i j}^{0}} \widehat{\theta}\right)\left.(k)\right|^{2} \\
& \leq \sum_{k \in \mathbb{Z}^{d}}\left|\widehat{\partial_{i} T_{i j}^{\nu}}\right|^{2}|\widehat{\phi}|^{2}(k)+\sum_{k \in \mathbb{Z}^{3}}\left|\widehat{\partial_{i} T_{i j}^{\nu}}-\widehat{\partial_{i} T_{i j}^{0}}\right|^{2}|\widehat{\theta}|^{2}(k) \\
& \leq \sum_{k \in \mathbb{Z}^{d}}\left|\widehat{T_{i j}^{\nu}}(k)\right|^{2}|\widehat{\nabla \phi}(k)|^{2}+\sum_{k \in \mathbb{Z}^{3}}\left|\widehat{T_{i j}^{\nu}}(k)-\widehat{T_{i j}^{0}}(k)\right|^{2}|\widehat{\nabla \theta}(k)|^{2} \\
& :=I_{1}+I_{2} .
\end{aligned}
$$

Using the condition (1.13), the term $I_{1}$ can be estimated by

$$
I_{1} \leq C_{*}^{2} \sum_{k \in \mathbb{Z}^{d}}|\widehat{\nabla \phi}(k)|^{2}=C_{*}^{2}\|\nabla \phi(t, \cdot)\|_{L^{2}}^{2} .
$$

For the term $I_{2}$, by Theorem 3.1, $\theta$ is smooth and $\|\nabla \theta(t, \cdot)\|_{L^{2}}<\infty$. So the condition (1.4) can be applied and we have

$$
\lim _{\nu \rightarrow 0} I_{2}=\lim _{\nu \rightarrow 0} \sum_{k \in \mathbb{Z}^{d}: k \neq 0}\left|\widehat{T_{i j}^{\nu}}(k)-\widehat{T_{i j}^{0}}(k)\right|^{2}|\widehat{\nabla \theta}(k)|^{2}=0 .
$$

We apply (4.22) on (4.21) to obtain

$$
\left\|\left(u^{\nu}-u\right)(t, \cdot)\right\|_{L^{2}}^{2} \leq C_{*}^{2}\|\nabla \phi(t, \cdot)\|_{L^{2}}^{2}+I_{2},
$$

and hence using (4.24) on (4.20),

$$
\left|-\int\left(u^{\nu}-u\right) \cdot \nabla \theta \cdot \phi(t, x) d x\right| \leq \frac{\kappa}{4}\|\nabla \phi(t, \cdot)\|_{L^{2}}^{2}+\frac{\kappa}{4 C_{*}^{2}} I_{2}+\frac{4 C_{*}^{2}}{\kappa}\|\phi(t, \cdot)\|_{L^{2}}^{2}\|\nabla \theta(t, \cdot)\|_{L^{\infty}}^{2} .
$$

Applying (4.25) on (4.19), using Grönwall's inequality, taking $\nu \rightarrow 0$ and using (4.23), for all $t>0$, we conclude that

$$
\lim _{\nu \rightarrow 0}\left\|\left(\theta^{\nu}-\theta\right)(t, \cdot)\right\|_{L^{2}}^{2}=\lim _{\nu \rightarrow 0}\|\phi(t, \cdot)\|_{L^{2}}^{2}=0 .
$$

Case 2: $s>0$. We apply the Gagliardo-Nirenberg interpolation inequality for homogeneous Sobolev space (2.3) to obtain, for $s>0$ and $t>0$,

$$
\left\|\left(\theta^{\nu}-\theta\right)(t, \cdot)\right\|_{H^{s}} \leq C\left\|\left(\theta^{\nu}-\theta\right)(t, \cdot)\right\|_{L^{2}}^{\gamma}\left\|\left(\theta^{\nu}-\theta\right)(t, \cdot)\right\|_{H^{s+1}}^{1-\gamma},
$$

where $\gamma \in(0,1)$ depends on $s$, and $C>0$ is a positive constant which depends on $d$ but is independent of $\nu$. Using the bounds (4.1) as in Theorem 4.1, the term $\left\|\left(\theta^{\nu}-\theta\right)(t, \cdot)\right\|_{H^{s+1}}^{1-\gamma}$ is bounded uniformly in $\nu$ for all $t \geq \tau$. Hence by taking $\nu \rightarrow 0$ and applying the $L^{2}$ convergence (4.26), we have

$$
\lim _{\nu \rightarrow 0}\left\|\left(\theta^{\nu}-\theta\right)(t, \cdot)\right\|_{H^{s}}=0 \text { for } t \geq \tau
$$


which finishes the proof of Theorem 1.2 .

\section{The MG Equations}

\subsection{The explicit symbol of the MG operator}

We now return to the magnetogeostrophic active scalar equation discussed in the introduction. Specifically, we are interested in the following active scalar equation in the domain $(0, \infty) \times \mathbb{T}^{3}=(0, \infty) \times$ $[0,2 \pi]^{3}$ (with periodic boundary conditions):

$$
\left\{\begin{array}{l}
\partial_{t} \theta^{\nu}+u^{\nu} \cdot \nabla \theta^{\nu}=\kappa \Delta \theta^{\nu}+S \\
u=M^{\nu}\left[\theta^{\nu}\right], \theta(0, x)=\theta_{0}(x)
\end{array}\right.
$$

via a Fourier multiplier operator $M^{\nu}$ which relates $u^{\nu}$ and $\theta^{\nu}$. More precisely,

$$
u_{j}^{\nu}=M_{j}^{\nu}\left[\theta^{\nu}\right]=\left(\widehat{M_{j}^{\nu}} \hat{\theta}^{\nu}\right)^{\vee}
$$

for $j \in\{1,2,3\}$. The explicit expression for the components of $\widehat{M}^{\nu}$ as functions of the Fourier variable $k=\left(k_{1}, k_{2}, k_{3}\right) \in \mathbb{Z}^{3}$ are obtained from the constitutive law (1.8) to give

$$
\begin{aligned}
& \widehat{M}_{1}^{\nu}(k)=\left[k_{2} k_{3}|k|^{2}-k_{1} k_{3}\left(k_{2}^{2}+\nu|k|^{4}\right)\right] D(k)^{-1}, \\
& \widehat{M}_{2}^{\nu}(k)=\left[-k_{1} k_{3}|k|^{2}-k_{2} k_{3}\left(k_{2}^{2}+\nu|k|^{4}\right)\right] D(k)^{-1}, \\
& \widehat{M}_{3}^{\nu}(k)=\left[\left(k_{1}^{2}+k_{2}^{2}\right)\left(k_{2}^{2}+\nu|k|^{4}\right)\right] D(k)^{-1},
\end{aligned}
$$

where

$$
D(k)=|k|^{2} k_{3}^{2}+\left(k_{2}^{2}+\nu|k|^{4}\right)^{2} .
$$

Here $\kappa>0$ and $\nu \geq 0$ are some diffusive constants, $\theta_{0}$ is the initial condition, and $S=S(x)$ is a given smooth function that represents the forcing of the system. Furthermore, we restrict the system (5.1) to the function spaces where all functions (including the forcing $S$ and initial data $\theta_{0}$ ) have zero mean with respect to $x_{3}$ (refer to section 4 of [24] for further discussion of this restriction).

Details of the singular behaviour of the Fourier multiplier symbols for the operator $M^{0}$ in certain regions of Fourier space are given in 24. More general issues concerning the ill-posedness and wellposedness of the unforced $\mathrm{MG}^{0}$ equation can be found in 21, [24, 25. In particular, it is to be noted that the $\mathrm{MG}^{0}$ with $\kappa>0$ is the so-called critical MG equation in the sense of the delicate balance between the nonlinear term and the dissipative term. Various critical active scalar equations such as surface quasi-geostrophic equation (SQG) have received considerable attention in the past decade because of the challenging nature of this delicate balance, [3], [13, [14, [15], [18, [20, [29], 30]. On the other hand, as we discussed in 23], the $\mathrm{MG}^{\nu}$ equation with $\nu>0$, where the symbol $\widehat{M}^{\nu}$ decays like $k^{-2}$, is a case where the dissipative term dominates the nonlinear term. In [23] it is shown that in the case of the $\mathrm{MG}^{\nu}$ equation with $\nu>0$ and $\kappa>0$, even for singular initial data, the global solution is instantaneously $C^{\infty}$-smoothed and satisfied classically for all $t>0$. With this dichotomy in mind, we seek to determine the long time behaviour of the forced critical $\mathrm{MG}^{0}$ equation through the "vanishing viscosity" limit of the $\mathrm{MG}^{\nu}$ equation.

\subsection{The MG equations in the class of drift-diffusion equations}

We will now show that the $\mathrm{MG}^{\nu}, \nu \geq 0$, equations satisfy the conditions of the general class of drift diffusion equations given by (1.11)-(1.14). We write

$$
u_{j}^{\nu}=M_{j}^{\nu}\left[\theta^{\nu}\right]=\partial_{i} T_{i j}^{\nu},
$$

where we have denoted

$$
T_{i j}^{\nu}=-\partial_{i}(-\Delta)^{-1} M_{j}^{\nu} \text { for } \nu \geq 0 .
$$

In order to show that conditions (1.11)-(1.14) are satisfied, we need the following lemmas for $\left\{T_{i j}^{\nu}\right\}_{\nu \geq 0}$ : 
Lemma 5.1. Let $T_{i j}^{\nu}, T_{i j}^{0}$ be as defined in (5.6)-(5.7) in terms of $M^{\nu}$ and $M^{0}$. There are constants $C_{1}, C_{2}>0$ independent of $\nu$ such that, for all $1 \leq i, j \leq 3$,

$$
\begin{gathered}
\sup _{\nu \in(0,1]} \sup _{\left\{k \in \mathbb{Z}^{3}: k \neq 0\right\}}\left|\widehat{T}_{i j}^{\nu}(k)\right| \leq \sup _{\nu \in(0,1]} \sup _{\left\{k \in \mathbb{Z}^{3}: k \neq 0\right\}} \frac{\left|\widehat{M}^{\nu}(k)\right|}{|k|} \leq C_{1}, \\
\sup _{\left\{k \in \mathbb{Z}^{3}: k \neq 0\right\}}\left|\widehat{T}_{i j}^{0}(k)\right| \leq \sup _{\left\{k \in \mathbb{Z}^{3}: k \neq 0\right\}} \frac{\left|\widehat{M}^{0}(k)\right|}{|k|} \leq C_{2} .
\end{gathered}
$$

Proof. The bound (5.9) follows from the discussion in (24, Section 4) and we omit the proof.

To show the bound (5.8), we only give the details for $\widehat{M}_{1}^{\nu}$ since the cases for $\widehat{M}_{2}^{\nu}$ and $\widehat{M}_{3}^{\nu}$ are almost identical.

To prove (5.8), we fix $\nu \in(0,1]$ and consider the following cases:

Case 1: $|k|>\nu^{-\frac{1}{2}}$. Then for each $k \in \mathbb{Z}^{3} /\{k=0\}$,

$$
\frac{\left|\widehat{M}_{1}^{\nu}(k)\right|}{|k|}=\frac{\left.\left|k_{2} k_{3}\right| k\right|^{2}-k_{1} k_{3}\left(k_{2}^{2}+\nu|k|^{4}\right) \mid}{|k|\left(|k|^{2} k_{3}^{2}+\left(k_{2}^{2}+\nu|k|^{4}\right)^{2}\right)} \text {. }
$$

Since $k \neq 0$, so $|k| \geq\left|k_{j}\right| \geq 1$ for $j=1,2,3$, in particular $|k|^{-1}<\nu^{\frac{1}{2}}$. Hence we obtain

$$
\begin{aligned}
\frac{\left|\widehat{M}_{1}^{\nu}(k)\right|}{|k|} & \leq \frac{\left|k_{2} k_{3}\right||k|^{2}}{|k|^{3} k_{3}^{2}}+\frac{\left|k_{1} k_{3}\right| k_{2}^{2}}{|k|^{3} k_{3}^{2}}+\frac{\nu\left|k_{1} k_{3}\right||k|^{4}}{\nu^{2}|k|^{8}} \\
& \leq \frac{1}{\left|k_{3}\right|}+\frac{1}{\left|k_{3}\right|}+\frac{1}{\nu|k|^{2}} \\
& \leq 2+\frac{\nu}{\nu}=3 .
\end{aligned}
$$

Case 2: $|k| \leq \nu^{-\frac{1}{2}}$. Then for each $k \in \mathbb{Z}^{3} /\{k=0\}$,

$$
\begin{aligned}
\frac{\left|\widehat{M}_{1}^{\nu}(k)\right|}{|k|} & \leq \frac{\left|k_{2} k_{3}\right||k|^{2}}{|k|^{3} k_{3}^{2}}+\frac{\left|k_{1} k_{3}\right| k_{2}^{2}}{|k|^{3} k_{3}^{2}}+\frac{\nu\left|k_{1} k_{3}\right||k|^{4}}{|k|^{3} k_{3}^{2}} \\
& \leq \frac{1}{\left|k_{3}\right|}+\frac{1}{\left|k_{3}\right|}+\frac{\nu|k|^{2}}{\left|k_{3}\right|} \\
& \leq 2+\nu \cdot\left(\nu^{-\frac{1}{2}}\right)^{2}=3 .
\end{aligned}
$$

Combining two cases, we have

$$
\sup _{\nu \in(0,1]} \sup _{\left\{k \in \mathbb{Z}^{3}: k \neq 0\right\}} \frac{\left|\widehat{M}_{1}^{\nu}(k)\right|}{|k|} \leq 3,
$$

and hence (5.8) holds for some $C_{1}>0$ independent of $\nu$.

Lemma 5.2. For each $L>0$,

$$
\lim _{\nu \rightarrow 0} \sup _{\left\{k \in \mathbb{Z}^{3}: k \neq 0,|k| \leq L\right\}} \frac{\left|\widehat{M}^{\nu}(k)-\widehat{M}^{0}(k)\right|}{|k|}=0
$$


Proof. Again we only give the details for $\widehat{M}_{1}^{\nu}$. We fix $L>0$, then for each $k \in \mathbb{Z}^{3} \backslash(\{k=0\}$ with $|k| \leq L$, we have

$$
\begin{aligned}
& \frac{\left|\widehat{M}_{1}^{\nu}(k)-\widehat{M}_{1}^{0}(k)\right|}{|k|} \\
& =\frac{\left.\left|-\nu k_{1} k_{3}^{3}\right| k\right|^{6}+\nu k_{1} k_{2}^{4} k_{3}|k|^{4}-\nu^{2} k_{2} k_{3}|k|^{10}+\nu^{2} k_{1} k_{2}^{2} k_{3}|k|^{8}-2 \nu k_{2}^{3} k_{3}|k|^{6} \mid}{\left(|k|^{2} k_{3}^{2}+\nu^{2}|k|^{8}+2 \nu|k|^{4} k_{2}^{2}+k_{2}^{4}\right)\left(k_{3}^{2}|k|^{2}+k_{2}^{4}\right)|k|} . \\
& \leq \frac{\nu\left|k_{1}\right|\left|k_{3}\right|^{3}|k|^{6}}{|k|^{5} k_{3}^{4}}+\frac{\nu\left|k_{1}\right| k_{2}^{4}\left|k_{3}\right||k|^{4}}{|k|^{5} k_{3}^{4}}+\frac{\nu^{2}\left|k_{2}\right|\left|k_{3}\right||k|^{10}}{|k|^{5} k_{3}^{4}}+\frac{\left.\nu^{2}\left|k_{1}\right| k_{2}^{2}\left|k_{3}\right| k\right|^{8}}{|k|^{5} k_{3}^{4}}+\frac{2 \nu\left|k_{2}\right|^{3}\left|k_{3}\right||k|^{6}}{|k|^{5} k_{3}^{4}} \\
& \leq \nu L^{10}+\nu L^{10}+\nu^{2} L^{12}+\nu^{2} L^{12}+2 \nu L^{10} .
\end{aligned}
$$

Hence

$$
\lim _{\nu \rightarrow 0} \sup _{\left\{k \in \mathbb{Z}^{3}: k \neq 0,|k| \leq L\right\}} \frac{\left|\widehat{M}_{1}^{\nu}(k)-\widehat{M}_{1}^{0}(k)\right|}{|k|}=0 .
$$

Lemma 5.3. Let $g$ be a function such that $\|\nabla g\|_{L^{2}}<\infty$. Then we have

$$
\lim _{\nu \rightarrow 0} \sum_{k \in \mathbb{Z}^{3}: k \neq 0} \frac{\left|\widehat{M}^{\nu}(k)-\widehat{M}^{0}(k)\right|^{2}|\widehat{\nabla g}(k)|^{2}}{|k|^{2}}=0
$$

Proof. Fix $g$ with $\|\nabla g\|_{L^{2}}<\infty$ and let $\varepsilon>0$ be given. Then $\sum_{k \in \mathbb{Z}^{3}}|\widehat{\nabla g}(k)|^{2}<\infty$, so there exists $L=L(\varepsilon)>0$ such that $\sum_{k \in \mathbb{Z}^{3},|k|>L}|\widehat{\nabla g}(k)|^{2}<\varepsilon$. Hence

$$
\begin{aligned}
& \sum_{k \in \mathbb{Z}^{3}: k \neq 0} \frac{\left|\widehat{M}^{\nu}(k)-\widehat{M}^{0}(k)\right|^{2}|\widehat{\nabla g}(k)|^{2}}{|k|^{2}} \\
= & \sum_{k \in \mathbb{Z}^{3}: k \neq 0,|k| \leq L} \frac{\left|\widehat{M}^{\nu}(k)-\widehat{M}^{0}(k)\right|^{2}|\widehat{\nabla g}(k)|^{2}}{|k|^{2}}+\sum_{k \in \mathbb{Z}^{3}: k \neq 0,|k|>L} \frac{\left(\left|\widehat{M}^{\nu}(k)\right|^{2}+\left|\widehat{M}^{0}(k)\right|^{2}\right)|\widehat{\nabla g}(k)|^{2}}{|k|^{2}} \\
\leq & \left(\sup _{\left\{k \in \mathbb{Z}^{3}: k \neq 0,|k| \leq L\right\}} \frac{\left|\widehat{M}^{\nu}(k)-\widehat{M}^{0}(k)\right|}{|k|}\right)^{2}\|\nabla g\|_{L^{2}}^{2}+\left(C_{1}+C_{2}\right) \varepsilon .
\end{aligned}
$$

Using Lemma 5.2 and taking $\nu \rightarrow 0$ on (5.12),

$$
\lim _{\nu \rightarrow 0} \sum_{k \in \mathbb{Z}^{3}: k \neq 0} \frac{\left|\widehat{M}^{\nu}(k)-\widehat{M}^{0}(k)\right|^{2}|\widehat{\nabla g}(k)|^{2}}{|k|^{2}} \leq\left(C_{1}+C_{2}\right) \varepsilon .
$$

Since $\varepsilon>0$ is arbitrary, (5.11) follows.

In view of Lemma [5.15.3, the sequence of operators $\left\{T_{i j}^{\nu}\right\}_{\nu \geq 0}$ given by (5.6)-(5.7) satisfy the conditions (1.11) and (1.13)-(1.14). Moreover, following the discussion given in 24] pp. 298-299, $\left\{T_{i j}^{\nu}\right\}_{\nu \geq 0}$ also satisfy (1.12). By Theorem 3.1 and Theorem 4.1, there exists classical solutions $\theta^{\nu}(t, x) \in$ $C^{\infty}\left((0, \infty) \times \mathbb{T}^{3}\right)$ of (5.1)-(5.5), evolving from $\theta_{0}$ which satisfy the uniform bounds (4.1). The abstract Theorem 1.1 and Theorem 1.2 may therefore be applied to the MG equations in order to obtain the convergence of smooth solutions, and hence we have proven:

Theorem 5.4. Let $\theta_{0} \in L^{2}, S \in C^{\infty}$ and $\kappa>0$ be given. There exists a classical solution $\theta^{\nu}(t, x) \in$ $C^{\infty}\left((0, \infty) \times \mathbb{T}^{3}\right)$ of (5.1)-(5.5), evolving from $\theta_{0}$ for all $\nu \geq 0$. 
Theorem 5.5. Let $\theta_{0} \in L^{2}, S \in C^{\infty}$ and $\kappa>0$ be given. Then if $\theta^{\nu}, \theta$ are $C^{\infty}$ smooth classical solutions of (5.1)-(5.5) for $\nu>0$ and $\nu=0$ respectively with initial data $\theta_{0}$, then given $\tau>0$, for all $s \geq 0$, we have

$$
\lim _{\nu \rightarrow 0}\left\|\left(\theta^{\nu}-\theta\right)(t, \cdot)\right\|_{H^{s}}=0
$$

whenever $t \geq \tau$.

\section{The Existence of a Global Attractor}

With the results of Theorems 5.4 and 5.5 in place, we define a weak solution to the $\mathrm{MG}^{0}$ equation which we call a "vanishing viscosity" solution. We use this concept to prove the existence of a compact global attractor in $L^{2}\left(\mathbb{T}^{3}\right)$ for the $\mathrm{MG}^{\nu}$ equations (5.1)-(5.5) including the critical equation where $\nu=0$. We further obtain the upper semicontinuity of the global attractor as $\nu$ vanishes. First, we define a class of solutions to (1.10) as follows.

Definition 6.1. A weak solution to (5.1)-(5.5) with $\nu=0$ is a function $\theta \in C_{w}\left([0, T] ; L^{2}\left(\mathbb{T}^{3}\right)\right)$ with zero spatial mean that satisfies (5.1) in a distributional sense. That is, for any $\phi \in C_{0}^{\infty}\left((0, T) \times \mathbb{T}^{3}\right)$,

$$
-\int_{0}^{T}\left\langle\theta, \phi_{t}\right\rangle d t-\int_{0}^{T}\langle u \theta, \nabla \phi\rangle d t+\kappa \int_{0}^{T}\langle\nabla \theta, \nabla \phi\rangle d t=\left\langle\theta_{0}, \phi(0, x)\right\rangle+\int_{0}^{T}\langle S, \phi\rangle d t
$$

where $u=\left.u\right|_{\nu=0}$. A weak solution $\theta(t)$ to (5.1) on $[0, T]$ with $\nu=0$ is called a "vanishing viscosity" solution if there exist sequences $\nu_{n} \rightarrow 0$ and $\left\{\theta^{\nu_{n}}\right\}$ such that $\left\{\theta^{\nu_{n}}\right\}$ are smooth solutions to (5.1) as given by Theorem 3.1 and $\theta^{\nu_{n}} \rightarrow \theta$ in $C_{w}\left([0, T] ; L^{2}\right)$ as $\nu_{n} \rightarrow 0$.

Remark 6.2. By Theorem 5.4 and Theorem 5.5, for any initial data $\theta_{0} \in L^{2}$, there exists a "vanishing viscosity" solution $\theta$ of (1.10) on $[0, \infty)$ with $\theta(0)=\theta_{0}$.

We prove that the equation (5.1) driven by a force $S$ possesses a compact global attractor in $L^{2}\left(\mathbb{T}^{3}\right)$ which is upper semicontinuous at $\nu=0$. More precisely, we have

Theorem 6.3. Assume $S \in C^{\infty}$. Then the system (5.1)-(5.5) with $\nu=0$ possesses a compact global attractor $\mathcal{A}$ in $L^{2}\left(\mathbb{T}^{3}\right)$, namely

$$
\mathcal{A}=\left\{\theta_{0}: \theta_{0}=\theta(0) \text { for some bounded complete "vanishing viscosity" solution } \theta(t)\right\} .
$$

For any bounded set $\mathcal{B} \subset L^{2}\left(\mathbb{T}^{3}\right)$, and for any $\varepsilon, T>0$, there exists $t_{0}$ such that for any $t_{1}>t_{0}$, every "vanishing viscosity" solution $\theta(t)$ with $\theta(0) \in \mathcal{B}$ satisfies

$$
\|\theta(t)-x(t)\|_{L^{2}}<\varepsilon, \forall t \in\left[t_{1}, t_{1}+T\right],
$$

for some complete trajectory $x(t)$ on the global attractor $(x(t) \in \mathcal{A}, \forall t \in(-\infty, \infty))$. Furthermore, for $\nu \in[0,1]$, there exists a compact global attractor $\mathcal{A}^{\nu} \subset L^{2}$ for (5.1) such that $\mathcal{A}^{0}=\mathcal{A}$ and $\mathcal{A}^{\nu}$ is upper semicontinuous at $\nu=0$, which means that

$$
\sup _{\phi \in \mathcal{A}^{\nu}} \inf _{\psi \in \mathcal{A}}\|\phi-\psi\|_{L^{2}} \rightarrow 0 \text { as } \nu \rightarrow 0 .
$$

Before we give the proof of Theorem 6.3, we state the following proposition. It gives an energy equality which is important in obtaining an absorbing ball for (5.1) (see Remark 6.6 below).

Proposition 6.4 (The energy equality). Let $\theta(t)$ be a "vanishing viscosity" solution of (5.1) on $[0, \infty)$ with $\theta(0) \in L^{2}$. Then $\theta(t)$ satisfies the following energy equality:

$$
\frac{1}{2}\|\theta(t)\|_{L^{2}}^{2}+\kappa \int_{t_{0}}^{t}\|\nabla \theta(s, \cdot)\|_{L^{2}}^{2} d s=\frac{1}{2}\left\|\theta\left(t_{0}\right)\right\|_{L^{2}}^{2}+\int_{t_{0}}^{t} \int_{\mathbb{T}^{3}} S \theta d x d s,
$$

for all $0 \leq t_{0} \leq t$ 
Proof. It suffices to show that the flux term $\int_{t_{0}}^{t} \int_{\mathbb{T}^{3}} u \theta \cdot \nabla \theta d x d s$ equals zero; for which a proof can be found in [8] and we omit the details. The proof uses techniques of Littlewood-Paley decomposition. It is analogous to the proof given in [7] that the energy flux is zero for weak solutions of the three dimensional Euler equation that are smoother than Onsager critical.

Remark 6.5. Based on the equality (6.2), we can see that every "vanishing viscosity" solution to (5.1) is strongly continuous in $t$.

Remark 6.6. Moreover, in view of (6.2), there exists an absorbing ball $\mathcal{Y}$ for (5.1) given by

$$
\mathcal{Y}=\left\{\theta \in L^{2}:\|\theta\|_{L^{2}} \leq R\right\}
$$

where $R$ is any number larger than $\kappa^{-1}\|S\|_{H^{-1}\left(\mathbb{T}^{3}\right)}$. Then for any bounded set $\mathcal{B} \subset L^{2}$, there exists a time $t_{0}$ such that

$$
\theta(t) \in \mathcal{Y}, \quad \forall t \geq t_{0},
$$

for every "vanishing viscosity" solution $\theta(t)$ with the initial data $\theta(0) \in \mathcal{B}$.

To facilitate the proof of Theorem 6.3 , we introduce the following notions:

- We denote the strong and weak distances on $L^{2}\left(\mathbb{T}^{3}\right)$ respectively by

$$
d_{s}(\phi, \psi)=\|\phi-\psi\|_{L^{2}} ; \quad d_{w}(\phi, \psi)=\sum_{k \in \mathbb{Z}^{3}} \frac{1}{2^{|k|}} \frac{\left|\hat{\phi}_{k}-\hat{\psi}_{k}\right|}{1+\left|\hat{\phi}_{k}-\hat{\psi}_{k}\right|},
$$

where $\hat{\phi}_{k}$ and $\hat{\psi}_{k}$ are the Fourier coefficients of $\phi$ and $\psi$.

. We let

$$
\mathcal{T}=\{I: I=[T, \infty) \subset \mathbb{R}, \text { or } I=(-\infty, \infty)\},
$$

and for each $I \subset \mathcal{T}$, let $\mathcal{F}(I)$ denote the set of all $\mathcal{Y}$-valued functions on $I$ (here $\mathcal{Y}$ is the absorbing ball given by (6.3) in Remark 6.6).

- We define $\pi^{\nu}: L^{2} \rightarrow L^{2}$ as the map $\pi^{\nu} \theta_{0}=\theta^{\nu}$, where $\theta^{\nu}$ is the solution to (5.1)-(5.5) given by Theorem 5.4.

We first prove the following lemma which gives the continuity of $\pi^{\nu}(t) \theta_{0}$ in $\nu$ for a given $\theta_{0}$. More precisely, we have:

Lemma 6.7. For $t>0, \pi^{\nu}(t) \theta_{0}$ is continuous in $\nu$, uniformly for $\theta_{0}$ in compact subsets of $L^{2}$.

Proof. We let $\mathcal{K}$ be a compact subset of $L^{2}$ and fix $\theta_{0} \in \mathcal{K}$. Let $\nu_{1}, \nu_{2} \in[0,1]$, then for each $t>0$, we have

$$
\frac{1}{2} \frac{d}{d t}\left\|\left(\theta^{\nu_{1}}-\theta^{\nu_{2}}\right)(t, \cdot)\right\|_{L^{2}}^{2}+\frac{\kappa}{2}\left\|\nabla\left(\theta^{\nu_{1}}-\theta^{\nu_{2}}\right)(t, \cdot)\right\|_{L^{2}}^{2}=-\int\left(u^{\nu_{1}}-u^{\nu_{2}}\right) \cdot \nabla \theta^{\nu_{2}} \cdot\left(\theta^{\nu_{1}}-\theta^{\nu_{2}}\right)(t, x) d x,
$$

where $\theta^{\nu_{i}}(0)=\theta_{0}$ for $i=1,2$. We follow the argument given in the proof of Theorem 1.2 to obtain

$$
\begin{aligned}
& \left|-\int\left(u^{\nu}-u\right) \cdot \nabla \theta^{\nu_{2}} \cdot\left(\theta^{\nu_{1}}-\theta^{\nu_{2}}\right)(t, x) d x\right| \\
& \leq \frac{\kappa}{4 C^{2}}\left\|\left(u^{\nu_{1}}-u^{\nu_{2}}\right)(t, \cdot)\right\|_{L^{2}}^{2}+\frac{4 C^{2}}{\kappa}\left\|\left(\theta^{\nu_{1}}-\theta^{\nu_{2}}\right)(t, \cdot)\right\|_{L^{2}}^{2}\left\|\nabla \theta^{\nu_{2}}(t, \cdot)\right\|_{L^{\infty}}^{2} .
\end{aligned}
$$

Using the bound (4.1) for $\theta^{\nu_{2}}$, the second term on the right side of (6.5) is bounded by $C\left\|\left(\theta^{\nu_{1}}-\theta^{\nu_{2}}\right)(t, \cdot)\right\|_{L^{2}}^{2}$, for some constant $C>0$ independent of $\theta_{0}$ but depends on the compact set $\mathcal{K}$ 
and $t$. On the other hand, the term $\left\|\left(u^{\nu_{1}}-u^{\nu_{2}}\right)(t, \cdot)\right\|_{L^{2}}^{2}$ can be bounded as follows.

$$
\begin{aligned}
\left\|\left(u^{\nu_{1}}-u^{\nu_{2}}\right)(t, \cdot)\right\|_{L^{2}}^{2} & \left.=\sum_{k \in \mathbb{Z}^{3}} \mid \widehat{\partial_{i} T_{i j}^{\nu_{1}}} \widehat{\theta^{\nu_{1}}}-\widehat{\partial_{i} T_{i j}^{\nu_{2}}} \widehat{\theta^{\nu_{2}}}\right)\left.(k)\right|^{2} \\
& \leq \sum_{k \in \mathbb{Z}^{3}}\left|\widehat{T_{i j}^{\nu_{1}}}(k)\right|^{2}\left|\nabla\left(\widehat{\theta^{\nu_{1}}-\theta^{\nu_{2}}}\right)(k)\right|^{2}+\sum_{k \in \mathbb{Z}^{3}}\left|\widehat{T_{i j}^{\nu_{1}}}(k)-\widehat{T_{i j}^{\nu_{2}}}(k)\right|^{2}\left|\widehat{\nabla \theta^{\nu_{2}}}(k)\right|^{2} \\
& \leq C^{2}\left\|\nabla\left(\theta^{\nu_{1}}-\theta^{\nu_{2}}\right)(t, \cdot)\right\|_{L^{2}}^{2}+\sum_{k \in \mathbb{Z}^{3}}\left|\widehat{T_{i j}^{\nu_{1}}}(k)-\widehat{T_{i j}^{\nu_{2}}}(k)\right|^{2}\left|\widehat{\nabla \theta^{\nu_{2}}}(k)\right|^{2},
\end{aligned}
$$

where $T_{i j}^{\nu_{1}}, T_{i j}^{\nu_{2}}$ are defined in (5.7). Using the bound (4.1) for $\theta^{\nu_{2}}$ again and applying the similar argument given in the proof of Lemma 5.1 and Lemma 5.2. there exists constant $C=C\left(\nu_{1}, \nu_{2}, \mathcal{K}, t\right)>0$ such that

$$
\sum_{k \in \mathbb{Z}^{3}}\left|\widehat{T_{i j}^{\nu_{1}}}(k)-\widehat{T_{i j}^{\nu_{2}}}(k)\right|^{2}\left|\widehat{\nabla \theta^{\nu_{2}}}(k)\right|^{2} \leq C\left|\nu_{1}-\nu_{2}\right| .
$$

Hence we conclude from (6.4) that

$$
\frac{1}{2} \frac{d}{d t}\left\|\left(\theta^{\nu_{1}}-\theta^{\nu_{2}}\right)(t, \cdot)\right\|_{L^{2}}^{2} \leq C\left[\left|\nu_{1}-\nu_{2}\right|+\left\|\left(\theta^{\nu_{1}}-\theta^{\nu_{2}}\right)(t, \cdot)\right\|_{L^{2}}^{2}\right] .
$$

Integrating the above over $t$ and using Grönwall's inequality, it further implies

$$
\begin{aligned}
\left\|\left(\pi^{\nu_{1}}(t) \theta_{0}-\pi^{\nu_{2}}(t) \theta_{0}\right)\right\|_{L^{2}}^{2}=\left\|\left(\theta^{\nu_{1}}-\theta^{\nu_{2}}\right)(t, \cdot)\right\|_{L^{2}}^{2} & \leq C e^{C t} \int_{0}^{t}\left|\nu_{1}-\nu_{2}\right| d s \\
& \leq C e^{C t} t\left|\nu_{1}-\nu_{2}\right|,
\end{aligned}
$$

hence we prove the continuity of $\pi^{\nu}$ in $\nu$ uniformly for $\theta_{0}$ in $\mathcal{K}$.

Next, the following lemma shows that the weak upper semicontinuity implies the strong upper semicontinuity.

Lemma 6.8. Let $\phi^{\nu_{j}} \in \mathcal{A}^{\nu_{j}}$ and $\psi_{j} \in \mathcal{A}$ be such that

$$
\lim _{j \rightarrow \infty} d_{w}\left(\phi^{\nu_{j}}, \psi_{j}\right)=0
$$

for some sequence $\nu_{j} \rightarrow 0$. Then

$$
\lim _{j \rightarrow 0}\left\|\phi^{\nu_{j}}-\psi_{j}\right\|_{L^{2}}=0 .
$$

Proof. Assume the conclusion of the lemma does not hold. Then passing to a subsequence and dropping a subindex, we can assume that

$$
\liminf _{j \rightarrow 0}\left\|\phi^{\nu_{j}}-\psi_{j}\right\|_{L^{2}}>0 .
$$

There are solutions $\theta_{j}^{\nu_{j}}(t, \cdot), \theta_{j}^{0}(t, \cdot)$ (with $\nu=\nu_{j}$ and $\nu=0$ respectively) which are complete bounded in $L^{2}$ such that $\theta_{j}^{\nu_{j}}(\cdot, 1)=\phi^{\nu_{j}}$ and $\theta_{j}^{0}(\cdot, 1)=\psi_{j}$.

Due to the energy equality (6.2) and the fact that the radius of the absorbing ball $\mathcal{Y}$ does not depend on $\nu$, there exists a constant $C>0$ independent of $\nu$ such that

$$
\int_{0}^{1}\left\|\nabla \theta_{j}^{\nu_{j}}(t, \cdot)\right\|_{L^{2}}^{2} d t \leq C, \quad \int_{0}^{1}\left\|\nabla \theta_{j}^{0}(t, \cdot)\right\|_{L^{2}}^{2} d t \leq C,
$$

for all $j$. This implies there exists a sequence $t_{j} \in[0,1]$ such that

$$
\left\|\nabla \theta_{j}^{\nu_{j}}\left(\cdot, t_{j}\right)\right\|_{L^{2}}^{2} \leq C, \quad\left\|\nabla \theta_{j}^{0}\left(\cdot, t_{j}\right)\right\|_{L^{2}}^{2} \leq C .
$$

Since the interval $[0,1]$ is compact, passing to a subsequence and dropping a subindex, we can assume that $t_{j} \rightarrow t^{*}$ for some $t^{*} \in[0,1]$. And using (6.8), by compactness, we can pass to another subsequence and drop a subindex to obtain

$$
\theta_{j}^{\nu_{j}}\left(\cdot, t_{j}\right) \rightarrow \bar{\theta}_{0}(\cdot), \theta_{j}^{0}\left(\cdot, t_{j}\right) \rightarrow \overline{\bar{\theta}}_{0}(\cdot) \text { in } L^{2},
$$


for some $\bar{\theta}_{0}, \overline{\bar{\theta}}_{0} \in L^{2}$.

For $\nu=0$, we consider solutions $\bar{\theta}$ and $\overline{\bar{\theta}}$ with $\bar{\theta}\left(\cdot, t^{*}\right)=\bar{\theta}_{0}$ and $\overline{\bar{\theta}}\left(\cdot, t^{*}\right)=\overline{\bar{\theta}}_{0}$. Note that we have $\theta^{\nu_{j}}\left(t_{j}\right) \rightarrow \bar{\theta}\left(t^{*}\right)$ in $L^{2}$ as $t_{j} \rightarrow t^{*}$. Following a similar proof of (6.6), there exists a constant $C=C\left(\bar{\theta}_{0}\right)>0$ independent of $\nu$ such that

$$
\lim _{j \rightarrow \infty}\left\|\theta_{j}^{\nu_{j}}(\cdot, 1)-\bar{\theta}(\cdot, 1)\right\|_{L^{2}} \leq \lim _{j \rightarrow \infty} C \int_{t^{*}}^{1} \nu_{j} d s=0 .
$$

Similarly, we also have

$$
\lim _{j \rightarrow \infty}\left\|\theta_{j}^{0}(\cdot, 1)-\overline{\bar{\theta}}(\cdot, 1)\right\|_{L^{2}}=0 .
$$

So we have shown that there is a sequence $j_{n}$ such that

$$
\lim _{n \rightarrow \infty}\left\|\phi^{\nu_{j_{n}}}(\cdot)-\bar{\theta}(\cdot, 1)\right\|_{L^{2}}=\lim _{n \rightarrow \infty} \psi_{j_{n}}(\cdot)-\overline{\bar{\theta}}(\cdot, 1) \|_{L^{2}}=0 .
$$

Hence $\bar{\theta}(\cdot, 1)=\overline{\bar{\theta}}(\cdot, 1)$ and we have $\left\|\phi^{\nu_{n}}-\psi_{j_{n}}\right\|_{L^{2}} \rightarrow 0$ as $n \rightarrow \infty$, which contradicts (6.7).

We are now ready to prove Theorem 6.3 .

Proof of Theorem 6.3. We define

$$
\begin{array}{r}
\mathcal{E}[T, \infty)=\{\theta(\cdot): \theta(\cdot) \text { is a "vanishing viscosity" solution of (1.10) } \\
\text { on }[T, \infty) \text { and } \theta \in \mathcal{Y} \text { for all } t \in[T, \infty)\}, \\
\mathcal{E}(-\infty, \infty)=\{\theta(\cdot): \theta(\cdot) \text { is a "vanishing viscosity" solution of (1.10) } \\
\text { on }(-\infty, \infty) \text { and } \theta \in \mathcal{Y} \text { for all } t \in(-\infty, \infty)\},
\end{array}
$$

then $\mathcal{E}$ is an evolutionary system (see [6] and [8] for the definition), so by Theorem 4.5 in [8], there exists a weak global attractor $\mathcal{A}_{w}$ to $\mathcal{E}$ with

$$
\mathcal{A}_{w}=\left\{\theta_{0}: \theta_{0}=\theta(0) \text { for some } \theta \in \mathcal{E}((-\infty, \infty))\right\} .
$$

Furthermore, by the Aubin-Lions Lemma (also refer to [11] for the case of Navier-Stokes equation), if $\theta_{n}(t)$ is any sequence of "vanishing viscosity" solutions of (1.10) such that $\theta_{n}(t) \in \mathcal{Y}$ for all $t \geq t_{0}$, then there exists a subsequence $\theta_{n_{j}}$ of $\theta_{n}$ that converges in $C\left(\left[t_{0}, T\right] ; \mathcal{Y}_{w}\right)$ to some "vanishing viscosity" solution $\theta(t)$ (here $\mathcal{Y}_{w}$ refers to the metric space $\left(\mathcal{Y}, d_{w}\right)$ ). Applying the arguments given in 8$], \mathcal{E}$ satisfies all the following properties:

A1 $\mathcal{E}([0, \infty))$ is a compact set in $C\left([0, \infty) ; \mathcal{Y}_{w}\right)\left(\mathcal{Y}_{w}\right.$ is endowed with the weak topology induced by $\left.d_{w}\right)$

A2 for any $\varepsilon>0$, there exists $\delta>0$ such that for every $\theta \in \mathcal{E}([0, \infty))$ and $t>0$,

$$
\|\theta(t)\|_{L^{2}} \leq\|\theta(t)\|_{L^{2}}+\varepsilon
$$

for $t_{0}$ a.e. in $(t-\delta, t) \cap[0, \infty)$;

A3 if $\theta_{n} \in \mathcal{E}([0, \infty))$ and $\theta_{n} \rightarrow \theta \in \mathcal{E}([0, \infty))$ in $C\left([0, \infty) ; \mathcal{Y}_{w}\right)$ for some $T>0$, then $\theta_{n}(t) \rightarrow \theta(t)$ strongly a.e. in $[0, T]$.

Therefore, together with Remark 6.6. Theorem 4.5 in $[8$ can then be applied again to our evolutionary system $\mathcal{E}$, which implies that

- the strong global attractor $\mathcal{A}_{s}$ exists, it is strongly compact and $\mathcal{A}:=\mathcal{A}_{s}=\mathcal{A}_{w}$; and

- for any bounded set $\mathcal{B} \subset L^{2}\left(\mathbb{T}^{3}\right)$, and for any $\varepsilon, T>0$, there exists $t_{0}$ such that for any $t_{1}>t_{0}$, every "vanishing viscosity" solution $\theta(t)$

$$
\|\theta(t)-x(t)\|_{L^{2}}<\varepsilon, \forall t \in\left[t_{1}, t_{1}+T\right],
$$

for some complete trajectory $x(t)$ on the global attractor $(x(t) \in \mathcal{A}, \forall t \in(-\infty, \infty))$. 
Finally, to prove that (6.1) holds, we recall that $\pi^{\nu}: L^{2} \rightarrow L^{2}$ is the map $\pi^{\nu} \theta_{0}=\theta^{\nu}$, where $\theta^{\nu}$ is the solution to (5.1) - (5.5) given by Theorem [5.4. Then for each $\nu \in(0,1], \pi^{\nu}$ is a semigroup $\left\{\pi^{\nu}(t)\right\}_{t \geq 0}$ on $L^{2}$. And using the above argument, there exists a compact global attractor $\mathcal{A}^{\nu} \subset L^{2}$ for $\pi^{\nu}$ given by

$$
\begin{aligned}
\mathcal{A}^{\nu}=\left\{\theta_{0}: \theta_{0}=\theta^{\nu}(0), \text { where } \theta^{\nu}\right. \text { is a solution of (5.1)-(5.5) } \\
\text { defined on } \left.(-\infty, \infty) \text { and } \theta^{\nu} \in \mathcal{Y} \text { for all } t \in(-\infty, \infty)\right\} .
\end{aligned}
$$

Moreover, for $\nu=0, \mathcal{A}^{0}=\mathcal{A}$ is the global attractor for $\pi^{0}(\cdot)$ satisfying

- $\pi^{0}(t) \mathcal{A}=\mathcal{A}$ for all $t \in \mathbb{R}$

- for any bounded set $\mathcal{B}, \sup _{\phi \in \pi^{0}(t) \mathcal{B}} \inf _{\psi \in \mathcal{A}}\|\phi-\psi\|_{L^{2}} \rightarrow 0$ as $t \rightarrow 0$.

We claim that the following conditions hold:

L1 $\pi_{\nu}(\cdot)$ has a global attractor $\mathcal{A}^{\nu}$ for every $\nu \in[0,1]$ in the weak- $L^{2}$ sense, which means that - $\pi^{0}(t) \mathcal{A}^{\nu}=\mathcal{A}^{\nu}$ for all $t \in \mathbb{R}$;

- for any bounded set $\mathcal{B}, \sup _{\phi \in \pi^{0}(t) \mathcal{B}} \inf _{\psi \in \mathcal{A}} d_{w}(\phi, \psi) \rightarrow 0$ as $t \rightarrow 0$.

L2 there is a compact subset $\mathcal{K}$ of $L^{2}$ in the weak topology induced by $d_{w}$ such that $\mathcal{A}^{\nu} \subset \mathcal{K}$ for every $\nu \in[0,1]$.

L3 for $t>0, \pi^{\nu}(t) \theta_{0}$ is continuous in $\nu$, uniformly for $\theta_{0}$ in compact subsets of $L^{2}$.

Notice that Lemma 6.7 implies L3, so we only have to show L1 and L2:

- To show L1, we note that the absorbing ball $\mathcal{Y}$ as given by (6.3) has radius which is independent of $\nu$, hence $\pi_{\nu}(\cdot)$ has a global attractor $\mathcal{A}^{\nu}$ for every $\nu \in[0,1]$ satisfying L1.

- To show $\mathrm{L} 2$, using $\mathrm{A} 1$, we have that $\mathcal{E}([0, \infty))$ is a compact set in $C\left([0, \infty) ; \mathcal{Y}_{w}\right)$, where $\mathcal{Y}_{w}$ refers to the metric space $\left(\mathcal{Y}, d_{w}\right)$ endowed with the weak topology induced by $d_{w}$. Hence we take $\mathcal{K}=\mathcal{Y}_{w}$ and $\mathcal{A}^{\nu} \subset \mathcal{K}$ for every $\nu \in[0,1]$.

In view of L1 to L3, the result from [27] implies the weak upper semicontinuity, namely

$$
\sup _{\phi \in \mathcal{A}^{\nu}} \inf _{\psi \in \mathcal{A}} d_{w}(\phi, \psi) \rightarrow 0 \text { as } \nu \rightarrow 0 .
$$

Hence using Lemma 6.8, (6.10) further implies the strong upper semicontinuity given by (6.1). This completes the proof of Theorem 6.3 .

\section{Acknowledgment}

We thank Vlad Vicol for his very helpful advice. We also thank the referees for their most valuable comments. A.S. is partially supported by Hong Kong Early Career Scheme (ECS) grant project number 28300016. S.F. is partially supported by NSF grant DMS-1613135.

\section{References}

[1] J. M. Aurnou et. al., Rotating convective turbulence in Earth and planetary cores, Phys of Earth and Planetary Interiors, 246, pp. 52-71, 2015.

[2] H. Bahouri, J. Chemin and R. Danchin, Fourier Analysis and Nonlinear Partial Differential Equations, Grundlehren der Mathematischen Wissenschaften 343 (Springer, 2011).

[3] L. Caffarelli, A. Vasseur, Drift diffusion equations with fractional diffusion and the quasi-geostrophic equation, Annals of Mathematics 171 (3), pp. 1903-1930, 2010.

[4] S. Chandrasekhar, The instability of a layer of fluid heated from below and subject to the simultaneous action of a magnetic field and rotation, Proc R. Soc London A Math Phys Sci, 225, pp. 173-184, 1954.

[5] Chen, Q., Miao, C., Zhang, Z., A new Bernstein's inequality and the 2D dissipative quasi-geostrophic equation, Comm. Math. Phys. 271(3), pp. 821-838, 2007.

[6] A. Cheskidov, Global attractors of evolutionary systems, Journal of Dynamics and Differential Equations, 21: pp. 249-268, 2009.

[7] A. Cheskidov, P. Constantin, S. Friedlander, and R. Shvydkoy, Energy conservation and Onsager?s conjecture for the Euler equations, Nonlinearity 21, pp. 1233-1252, 2008. 
[8] A. Cheskidov and M. Dai, The existence of a global attractor for the forced critical surface quasi-geostrophic equation in $L^{2}$, Journal of Mathematical Fluid Mechanics, DOI: 10.1007/s00021-017-0324-7, 2017.

[9] A. Cheskidov and Foias, On global attractors of the 3D Navier-Stokes equations, J. Differential Equations, 231(2), pp. 714-754, 2006.

[10] P. Constantin, M. Coti-Zelati, and V. Vicol, Uniformly attracting limit sets for the critically dissipative SQG equation, Nonlinearity, 29, pp. 298-318, 2016.

[11] P. Constantin and C. Foias, Navier-Stokes Equation, University of Chicago Press, Chicago, 1989.

[12] P. Constantin, A. Tarfulea, and V. Vicol, Absence of anomalous dissipation of energy in forced two dimensional fluid equations, Arch. Ration. Mech. Anal. 212, pp. 875-903, 2014.

[13] P. Constantin, A. Tarfulea, and V. Vicol, Long time dynamics of forced critical SQG, Comm. Math. Phys. 335, pp. 93-141, 2014.

[14] P. Constantin and V. Vicol, Nonlinear maximum principles for dissipative linear nonlocal operators and applications, Geom. Funct. Anal. 22, pp. 1289-1321, 2012.

[15] P. Constantin, J. Wu, Hölder continuity of solutions of supercritical dissipative hydrodynamic transport equations, Ann. Inst. H. Poincaré Anal. Non Linaire 26 (1), pp. 159-180, 2009.

[16] R. Danchin, Fourier Analysis Methods for PDEs. Lecture Notes, 14 November, 2005.

[17] P. A. Davidson, An Introduction to Magnetohydrodynamics, Cambridge Texts in Applied Math, Cambridge University Press, 2001

[18] H. Dong, Dissipative quasi-geostrophic equations in critical Sobolev spaces: smoothing effect and global well-posedness, Discrete Contin. Dyn. Syst. 26, pp. 1197-1211, 2010.

[19] J. Foldes, S. Friedlander, N. Glatt-Holtz and G. Richards, Asymptotic analysis for randomly forced MHD, SIAM Math Analysis, 49(6), pp. 4440-4469, 2017.

[20] S. Friedlander, N. Pavlović, and V. Vicol, Nonlinear instability for the critically dissipative quasigeostrophic equation, Comm. Math. Phys. 292, pp. 797-810, 2009.

[21] S. Friedlander, W. Rusin, and V. Vicol, On the supercritically diffusive magneto-geostrophic equations, Nonlinearity, 25(11): pp. 3071-3097, 2012.

[22] S. Friedlander, W. Rusin and V. Vicol, The magneto-geostrophic equations: a survey, Proceedings of the St. Petersburg Mathematical Society, Volume XV: Advances in Mathematical Analysis of Partial Differential Equations. (2014) D. Apushkinskaya, and A.I. Nazarov, eds. pp. 53-78.

[23] S. Friedlander and A. Suen, Existence, uniqueness, regularity and instability results for the viscous magneto-geostrophic equation, Nonlinearity, 28 (9), pp. 3193-3217, 2015.

[24] S. Friedlander and V. Vicol, Global well-posedness for an advection-diffusion equation arising in magnetogeostrophic dynamics, Ann. Inst. H. Poincaré Anal. Non Linéaire, 28(2): pp. 283-301, 2011.

[25] S. Friedlander and V. Vicol, On the ill/well-posedness and nonlinear instability of the magneto-geostrophic equations, Nonlinearity, 24(11): pp. 3019-3042, 2011.

[26] S. Friedlander and V. Vicol, Higher regularity of Hölder continuous solutions of parabolic equations with singular drift velocities, J. Math. Fluid Mech., 14(2): pp. 255-266, 2012.

[27] L.T. Hoang, E.J. Olson, and J.C. Robinson, On the continuity of global attractors, Proc. Amer. Math. Soc., 143:10 4389-4395, 2015.

[28] E. M. King and J. M. Aurnou, Magnetostrophic balance as the optimal state for turbulent magneto convection, PNAS, 112 no 4, pp. 990-994, 2015.

[29] A. Kiselev and F. Nazarov, A variation on a theme of Caffarelli and Vasseur, Zap. Nauchn. Sem. S.Peterburg. Otdel. Mat. Inst. Steklov. (POMI) 370 (2009), pp. 58-72, 220.

[30] A. Kiselev, F. Nazarov, and A. Volberg, Global well-posedness for the critical $2 D$ dissipative quasigeostrophic equation, Invent. Math. 167, pp. 445-453, 2007.

[31] P.G. Lemarié-Rieusset; Recent developments in the Navier-Stokes problem, Research Notes in Mathematics, Chapman \& Hall, CRC, 2002.

[32] G.M. Lieberman, Second Order Parabolic Differential Equations, World Scientific Publishing Co., River Edge, NJ, 1996.

[33] H.K. Moffatt and D.E. Loper, The magnetostrophic rise of a buoyant parcel in the earth's core, Geophysical Journal International, 117(2): pp. 394-402, 1994. 
[34] H.K. Moffatt, Magnetic Field Generation in Electrically Conducting Fluids, Cambridge Monographs on Mechanics, Cambridge University Press, 1978.

[35] H.K. Moffatt, Magnetostrophic turbulence and the geodynamo, In Y. Kaneda, editor, IUTAM Symposium on Computational Physics and New Perspectives in Turbulence, Nagoya, Japan, September, 11-14, 2006, volume 4 of IUTAM Bookser., pp. 339-346. Springer, Dordrecht, 2008.

[36] P. H. Roberts and E.M. King, On the genesis of the Earth's magnetism, Rep Prog Phys, 76 no 9, 2013

[37] Y.A. Semenov, Regularity theorems for parabolic equations, J. Funct. Anal. 231 (2) pp. 375-417, 2006.

Susan Friedlander

Department of Mathematics

University of Southern California

e-mail: susanfri@usc.edu

Anthony Suen

Department of Mathematics and Information Technology

The Education University of Hong Kong

e-mail: acksuen@eduhk.hk 